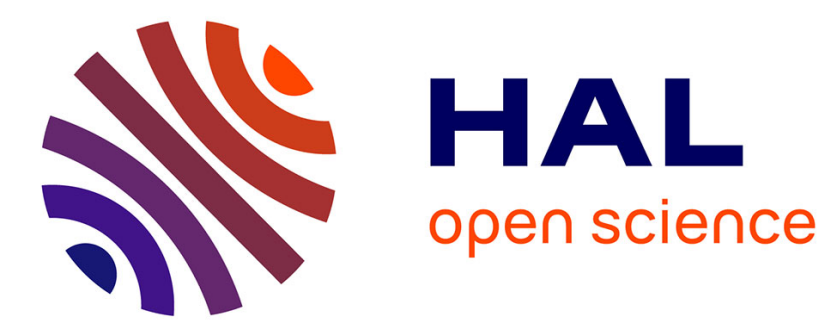

\title{
Stable and unstable monopolar vortices in a stratified fluid
}

\author{
J. Flór, J. van Heijst
}

\section{To cite this version:}

J. Flór, J. van Heijst. Stable and unstable monopolar vortices in a stratified fluid. Journal of Fluid Mechanics, 1996, 311 (1), pp.257-287. 10.1017/S0022112096002595 . hal-02140387

\section{HAL Id: hal-02140387 \\ https://hal.science/hal-02140387}

Submitted on 22 Sep 2020

HAL is a multi-disciplinary open access archive for the deposit and dissemination of scientific research documents, whether they are published or not. The documents may come from teaching and research institutions in France or abroad, or from public or private research centers.
L'archive ouverte pluridisciplinaire HAL, est destinée au dépôt et à la diffusion de documents scientifiques de niveau recherche, publiés ou non, émanant des établissements d'enseignement et de recherche français ou étrangers, des laboratoires publics ou privés. 


\title{
Stable and unstable monopolar vortices in a stratified fluid
}

\author{
By J. B. FLÓR† AND G. J. F. VAN HEIJST \\ Fluid Dynamics Laboratory, Department of Technical Physics, Eindhoven University of \\ Technology, PO Box 513, 5600MB Eindhoven, The Netherlands
}

This paper presents experiments on planar monopolar vortex structures generated in a non-rotating, stratified fluid. In order to study the dynamics of such planar vortices in the laboratory, angular momentum was generated in a specific horizontal layer of the stratified fluid, by using three different generation mechanisms. The lens-shaped monopolar vortices thus created were in some cases stable and conserved their circular symmetry, while in other cases they appeared to be unstable, leading to the formation of a multipoled vortex with a different topology. Characteristics such as cross-sectional profiles (angular velocity and vorticity) and vorticity-stream function scatter plots have been measured experimentally by using digital image processing techniques. The characteristics of the monopolar vortices are compared with analytical vortex models known from literature. Simple models, based on vertical diffusion of vorticity, are proposed to describe the monopolar vortex decay; they show reasonable agreement with the experimental results.

From the multipolar structures, the tripolar vortex and a specific case of a triangular vortex, neither having been observed before in a stratified fluid, are studied in detail. A comparison with point-vortex models yields good agreement. Although these multipolar vortices appear to persist for a long while, they are found eventually to be unstable and to transform into a monopolar vortex.

\section{Introduction}

At present it is well known that coherent vortex structures are common features of quasi-two-dimensional flows. For example, vortex structures have been detected on a range of scales in geophysical flows which are quasi-two-dimensional due to stratification, Earth's rotation and/or the geometry of the flow (the atmosphere and oceans are relatively thin shells around the Earth). Most well known are large-scale vortices (of 10 to $100 \mathrm{~km}$ in diameter), such as Gulf Stream Rings or Agulhas Rings, that have been observed by satellite imagery in the upper ocean, and large-scale vortex lenses, such as for instance Meddies (Mediterranean eddies) the existence of which has been indicated by in situ measurements in the deep ocean. The stability as well as the longevity of coherent vortices has motivated various scientists to study the dynamics of such vortices numerically, theoretically and in the laboratory.

Laboratory experiments on the stability of two-layer baroclinic monopolar vortices in a rotating fluid have been performed by Saunders (1973) and by Griffiths \&

$\uparrow$ Present address: University of Cambridge, Department of Applied Mathematics and Theoretical Physics, Silver Street, Cambridge CB3 9EW, UK. 
Linden (1981). The latter authors generated a homogeneous dome-shaped vortex by continuously adding or releasing a fixed volume of fluid of a higher (lower) density at the bottom (surface) of a rigidly rotating fluid. In some cases mode- 2 instabilities were observed to grow, eventually leading to the formation of two smaller monopoles or dipoles, while in other cases the flow relaxed again to a circularly symmetric vortex. The vortices created at the bottom of the tank (with fluid of a higher density than the tank fluid) appeared to be unstable and were seen to split up into smaller monopoles or dipoles, similar to the observations by Saunders (1973). Hedström \& Armi (1988) studied homogeneous lenses in a linearly stratified, rotating environment and these persisted for more than 150 background rotation periods. Since there was no internal stratification within the lens to suppress Ekman circulation, these vortices spun down mainly due to friction in the upper and lower boundaries of the vortex lens.

Stable and unstable barotropic monopolar vortices in a rotating homogeneous fluid have been investigated by Kloosterziel \& van Heijst $(1991,1992)$. Experimentally as well as theoretically, the effects of advection of relative vorticity, lateral diffusion and surface perturbations on the vortex velocity profile were studied and it was found that the advection of relative vorticity by Ekman circulation was mainly responsible for a gradual change of the vortex's azimuthal velocity profiles. Because this advection generally leads to a steepening of the velocity profile, it provides an important ingredient for the onset of instabilities. The vortices were unstable due to two-dimensional shearing instabilities as well as (three-dimensional) centrifugal instabilities, of which mainly the former were found to result in the formation of multipoled vortex structures. Unstable cyclonic vortices were observed to transform into a tripolar vortex, while some anticyclonic vortices ended up in a triangular vortex consisting of a triangular core and three satellite vortices. In some cases the triangular vortex was observed to split up into two vortex dipoles (see Kloosterziel \& van Heijst 1991), while more stable triangular vortices have been studied numerically and experimentally by Carnevale \& Kloosterziel (1994). Tripolar vortices were investigated in great detail by van Heijst, Kloosterziel \& Williams (1991). A general review of vortices in rotating fluids is given by Hopfinger \& van Heijst (1993).

Numerical simulations by Benzi, Patarnello \& Santangelo (1987) have revealed that the tripolar vortex may arise as a coherent structure in a purely two-dimensional, randomly initialized, turbulent flow field. Carton, Flierl \& Polvani (1989) and Orlandi \& van Heijst (1992) have performed numerical simulations of a single unstable monopolar vortex and they found an evolution to a stable tripolar vortex similar to the observations by van Heijst \& Kloosterziel (1989) and van Heijst et al. (1991).

The evolution of monopolar vortices in a non-rotating, stratified fluid and the formation as well as the stability of multipoled vortex structures with non-zero angular momentum have not been reported before. For this reason a systematic experimental study of monopolar vortices in a stratified fluid was undertaken. In contrast to largescale geostrophic vortices, such vortices are governed by a cyclostrophic balance, i.e. between nonlinear acceleration terms and pressure gradients, and their application should be found in small-scale geophysical flows for which the eddy-turnover time is much smaller than the Earth's rotation time.

Three essentially different generation methods for planar vortices were used: (i) a small sphere spinning about the vertical axis, which brings the ambient fluid into rotation; (ii) a rotating bent rod that locally stirs the fluid; and (iii) horizontal, tangential fluid injection along the inner wall of a thin-walled open cylinder that is placed in the stratified fluid with its axis vertical, by which a rotational motion within this cylinder is induced. In any of these cases, after lifting the forcing device a planar 


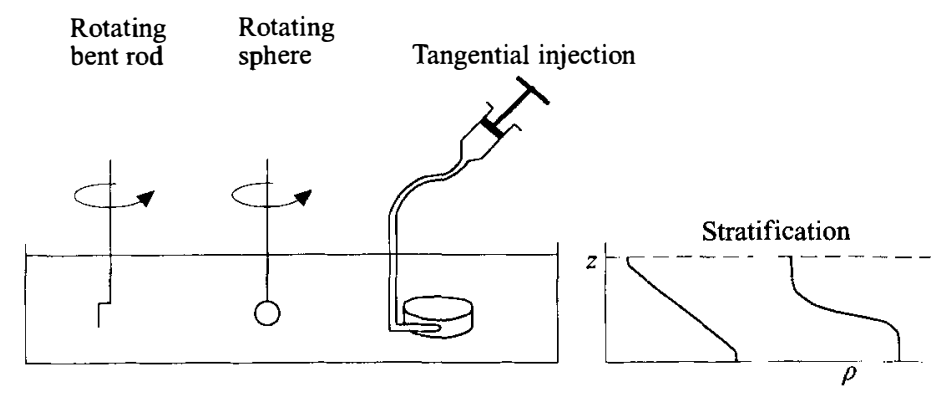

FIGURE 1. A schematic drawing of the experimental setup and the devices used for the generation of planar monopolar vortices.

vortex was created at half-depth in the otherwise quiescent stratified fluid. A total of 60 experiments were carried out, in which forcing and stratification parameters were varied systematically.

The remainder of this paper is organized as follows. After a description of the generation methods of monopolar vortices in $\S 2$, the characteristic properties of a stable monopolar vortex in a stratified fluid are considered in $\S 3$. The decay is modelled by using three simple decay models that are based on vertical diffusion. In the same section, a comparison with the experimental data is made. In $\S 4$ monopolar instabilities and the evolution of stable and unstable tripolar vortices will be discussed on the basis of experimental observations. Because the occurrence of the tripolar vortex and the triangular vortex in a stratified fluid has never been reported before, their characteristics have been investigated here in detail and the results are presented in $\S 5$; in $\S 6$ both types of vortex structures are compared with point-vortex models. In $\S 7$ the main conclusions are summarized and discussed.

\section{The generation of monopolar vortices}

\subsection{Experimental arrangement}

The laboratory experiments were carried out in a rectangular tank (of horizontal dimensions $115 \times 90 \mathrm{~cm}$ and $30 \mathrm{~cm}$ depth). For a number of experiments the tank was filled with fluid with a linear density stratification, which was established by the wellknown two-tank method (Fortuin 1960). In other cases the tank was filled with two fluids to form a stably stratified two-layer fluid, the thick diffused interface between the two layers being used for the experiments. The vortex motion was induced with different forcing devices (see figure 1), i.e. by a solid sphere (diameters $2.5 \mathrm{~cm}$ and $2.8 \mathrm{~cm}$ ), by a thin bent rod (diameter $0.25 \mathrm{~cm}$ ) with a vertical part of $4.5 \mathrm{~cm}$ and a radial arm of $3.0 \mathrm{~cm}$ (henceforth referred to as the stirrer), both rotating at a constant angular velocity about a vertical axis. A third generation technique consisted of horizontally injecting fluid along the inner wall of an open thin-walled cylinder that was placed in the stratified fluid, with its axis vertical (see figure 1). The injection fluid was prepared to have the same density as the ambient fluid at the level of injection and was carefully injected (by hand) so that the motion remained confined within the cylinder. After the injection was stopped, the turbulence was allowed to collapse and to evolve into a regular rotational motion inside the cylindrical housing (this took typically $10 \mathrm{~s}$ ), after which the device was carefully lifted out. This vortex generation technique is henceforth referred to as the tangential injection method. Two different cylindrical housings were used, one of $12 \mathrm{~cm}$ in diameter and a height of $3 \mathrm{~cm}$, and 

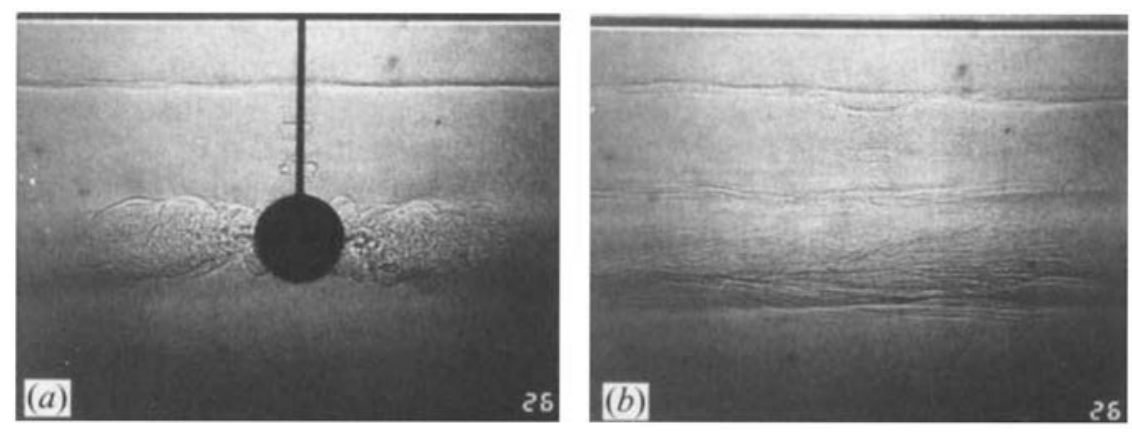

FIGURE 2. Shadowgraph visualization of the flow generated by a rotating sphere $(a)$ during forcing and $(b)$ after withdrawal. Experimental parameters: forcing rotation rate 675 r.p.m., $\delta t \approx 60 \mathrm{~s}$, linear stratification with $N=1.11 \mathrm{rad} \mathrm{s}^{-1}$, sphere diameter $3.8 \mathrm{~cm}$.

one of $15 \mathrm{~cm}$ in diameter and a height of $5 \mathrm{~cm}$. The injection nozzle was constructed flush to the inner cylinder wall. In both cases the nozzle diameter was $0.6 \mathrm{~cm}$ and the injection rate was varied from $Q=6$ to $28 \mathrm{ml} \mathrm{s}^{-1}$, yielding an outflow with a Reynolds number varying from 250 to 1500 . The fluid was injected for approximately $25 \mathrm{~s}$. The buoyancy frequency $N$ of the stratification, defined by $N=(-g / \rho \partial \rho / \partial z)^{1 / 2}$, was varied in these experiments between $N=1.8$ and $5.8 \mathrm{rad} \mathrm{s}^{-1}$. In the case of a two-layer fluid the gradient $\partial \rho / \partial z$ was averaged over the interface thickness (of typically $8 \mathrm{~cm}$ ).

In all experiments the forcing device was positioned at the mid-level of the interface in the case of a two-layer fluid or at mid-depth in the case of a linearly stratified fluid, and after the forcing was stopped the device was removed by carefully lifting it out. The horizontal vortex motion thus generated was confined in a thin layer of fluid, of typically $5 \mathrm{~cm}$ thickness.

The flow was visualized by addition of tracer particles to the fluid. Quantitative information about the horizontal flow field was obtained by applying a digital image analysis technique. After digitization of the particle streak pictures, the velocity vectors were interpolated to a rectangular grid of $30 \times 30$ grid points covering (part of) the flow domain. As a next step, the values of the vertical component of the vorticity $\omega=\partial v / \partial x-\partial u / \partial y$, with $v=(u, v)$ the velocity components in the horizontal $(x, y)$ directions, and the stream function $\psi$ (defined by $\boldsymbol{v}=\nabla \times \boldsymbol{k} \psi$, with $\boldsymbol{k}$ the unit vector in vertical direction) were calculated in each grid point (for a detailed description see Nguyen Duc \& Sommeria 1988 or Flór \& van Heijst 1994). In addition, plan-view dye visualizations were made to follow the vortical flow evolution qualitatively and side-view shadowgraph visualizations were made to study the evolution of the density field. The evolution of the density field is discussed below for the forcing by the rotating sphere and the stirrer.

\subsection{The flow induced by the stirring devices}

Figure $2(a)$ displays a shadowgraph visualization of the flow in the vicinity of the rotating sphere in a linearly stratified fluid. In the thin Ekman-type boundary layers on the sphere's upper and lower surfaces, fluid is accelerated and transported radially downwards and upwards to its equator, where it is swept radially outwards, inducing a turbulent mixing motion. The mixed fluid intrudes radially into the quiescent ambient fluid, and the intrusion proceeds as long as the flow is forced (see figure $2 a$ ).

After the sphere was withdrawn carefully, the turbulent motions collapsed and a laminar vortex formed at the level of the sphere equator. This vortex is confined 

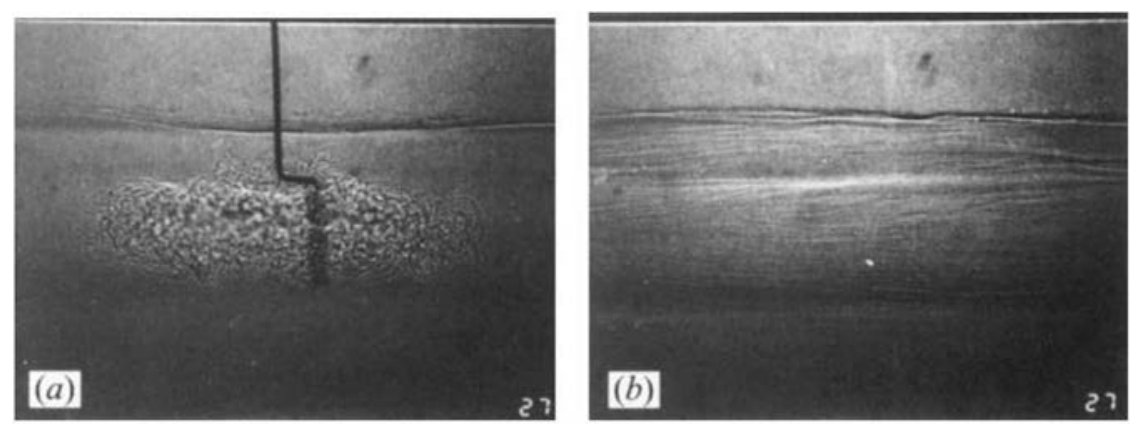

FIGURE 3. Shadowgraph visualization of the flow generated by the stirrer $(a)$ during forcing and $(b)$ after withdrawing the device. Experimental parameters: forcing rotation rate 150 r.p.m., $\delta t \approx 60 \mathrm{~s}$, linear stratification with $N=1.11 \mathrm{rad} \mathrm{s}^{-1}$. Dimensions of the stirrer (diameter $0.25 \mathrm{~cm}$ ): radial arm $2.5 \mathrm{~cm}$ and vertical part $4 \mathrm{~cm}$.

in a horizontal layer with a thickness of approximately the sphere diameter. The intrusion of mixed fluid has left two density interfaces, which are still visible as dark lines in the shadowgraphs, shown in figure $2(b)$. The vortex thus generated shows in many cases horizontal size oscillations, owing to internal wave motions that are generated by the lifting of the sphere. These internal waves remain trapped at the interfacial regions and usually persisted for a relatively long time after the forcing was stopped.

A shadowgraph visualization of the vortical flow generated by the stirrer in a linearly stratified fluid is shown in figure $3(a)$. Soon after starting the forcing a relatively large mixed region is formed. Top-view particle visualizations in an identical experiment revealed a monopolar vortex only a few seconds after the forcing is stopped. Although vertical motions are quickly damped by gravity, to some extent internal waves generated by the collapse are quite persistent and perturb the horizontal vortex motion (see figure $3 b$ ). Owing to these intense internal wave motions the monopolar vortex was hardly perceivable in some cases and, instead, the instantaneous formation of a vortex of a higher mode was observed. A monopolar vortex was only observed to form for slow rotation speeds.

The net circulation of the flow induced by the tangential-injection technique is zero, so that initially an isolated vortex will be generated. In contrast to the other forcing methods, the internal wave motions generated by the tangential injection were damped out within the cylinder region before the device was lifted out, and also the internal waves and the mixing caused by lifting the device were negligible compared to that of the other generation mechanisms. This forcing method was used for the dye visualizations of the evolution of unstable monopoles in particular.

\section{Stable monopolar vortices}

\subsection{Dynamics}

For the theoretical description of the present planar monopolar vortices one can apply the same scaling as Riley, Metcalfe \& Weissman (1981), which was also used by Flór \& van Heijst (1994) for the scaling of dipolar vortex motion in a stratified fluid. Applying a similar scaling on an axisymmetric vortex one obtains for the leading-order equations 


$$
\begin{aligned}
-\frac{v_{\theta}^{2}}{r} & =-\frac{1}{\bar{\rho}} \frac{\partial p^{\prime}}{\partial r} \\
\frac{\partial v_{\theta}}{\partial t} & =v\left(\nabla^{2} v_{\theta}-\frac{v_{\theta}}{r^{2}}+\frac{\partial^{2} v_{\theta}}{\partial z^{2}}\right), \\
0 & =-\frac{1}{\bar{\rho}} \frac{\partial p^{\prime}}{\partial z}-g^{\prime} \\
\frac{\partial \rho^{\prime}}{\partial t}+w \frac{\mathrm{d} \rho_{0}}{\mathrm{~d} z} & =0
\end{aligned}
$$

where $p^{\prime}$ denotes the pressure deviation; $\bar{\rho}=\rho\left(z_{0}\right)$ the constant reference density with $\rho^{\prime}$ the deviation from the ambient density $\rho_{0}=\rho(z)$, and $g^{\prime}=g \rho^{\prime} / \bar{\rho}$ the reduced gravity. As is apparent from equation $(3.1 a)$ the centrifugal force is balanced by the horizontal pressure gradient, implying a cyclostrophic balance, while in the vertical the flow is in hydrostatic balance $(3.1 c)$. Vertical motions are - to leading order decoupled from the horizontal flow, implying a quasi-two-dimensional flow.

Since the vortex motion is confined to a thin layer, shear is generated in the regions below and above the mid-plane. Therefore, the vorticity vector $\omega=\left(\omega_{r}, \omega_{\theta}, \omega_{z}\right)$ will be directed vertically only at the level $z=0$ where $\partial v_{\theta} / \partial z=0$, while for $|z|>0$ the horizontal vorticity associated with the shear $\partial v_{\theta} / \partial z$ is non-zero. The radial vorticity component $\omega_{r}$ can be derived by differentiation of $(3.1 b)$ with respect to $z$; apparently its evolution is governed purely by diffusion. By cross-differentation of $(3.1 a)$ and (3.1 c) with respect to $z$ and $r$, respectively, one obtains

$$
-2 \frac{v_{\theta}}{r} \frac{\partial v_{\theta}}{\partial z}=\frac{g}{\bar{\rho}} \frac{\partial \rho^{\prime}}{\partial r}
$$

showing that the shear is balanced by gravity as in the 'thermal wind' balance in rotating stratified systems. By scaling this equation with the maximum azimuthal velocity $V_{\max }$ at radius $R_{v \max }$ and the buoyancy frequency $N$ one can derive the relation

$$
h=\sqrt{2} \frac{V_{\max }}{N}
$$

where $h$ is a measure of the elevation of the isopycnals from their equilibrium position in the fluid at rest. Equation (3.3) represents a measure of the ratio between the pressure anomaly in the vortex centre due to the centrifugal force and the restoring buoyancy force. For a typical vortex with maximum velocity $V_{\max }=0.68 \mathrm{~cm} \mathrm{~s}^{-1}$ and $N=2.3 \mathrm{rad} \mathrm{s}^{-1}$ (see figure 4), the elevation of the isopycnals is of the order $O(4 \mathrm{~mm})$. This elevation is at most $8 \%$ of the initial vortex thickness (of $5 \mathrm{~cm}$ ) and decreases during the flow evolution by the exponential decay in the azimuthal velocity and the increase in vortex thickness. Since the maximum experimental error in the data is approximately $10 \%$, this effect becomes relatively small during the flow evolution and is therefore neglected in the data analysis.

For the vertical vorticity component $\omega_{z}$ one obtains (leaving the assumption of axisymmetry aside)

$$
\frac{\partial \omega_{z}}{\partial t}+J\left(\omega_{z}, \psi\right)=\frac{1}{R e}\left(\nabla_{h}^{2} \omega_{z}+\frac{1}{\beta^{2}} \frac{\partial^{2} \omega_{z}}{\partial z^{2}}\right)
$$

where $J$ is the Jacobian defined by

$$
J\left(\omega_{z}, \psi\right)=\frac{\partial \omega_{z}}{\partial r} \frac{1}{r} \frac{\partial \psi}{\partial \theta}-\frac{1}{r} \frac{\partial \omega_{z}}{\partial \theta} \frac{\partial \psi}{\partial r} .
$$




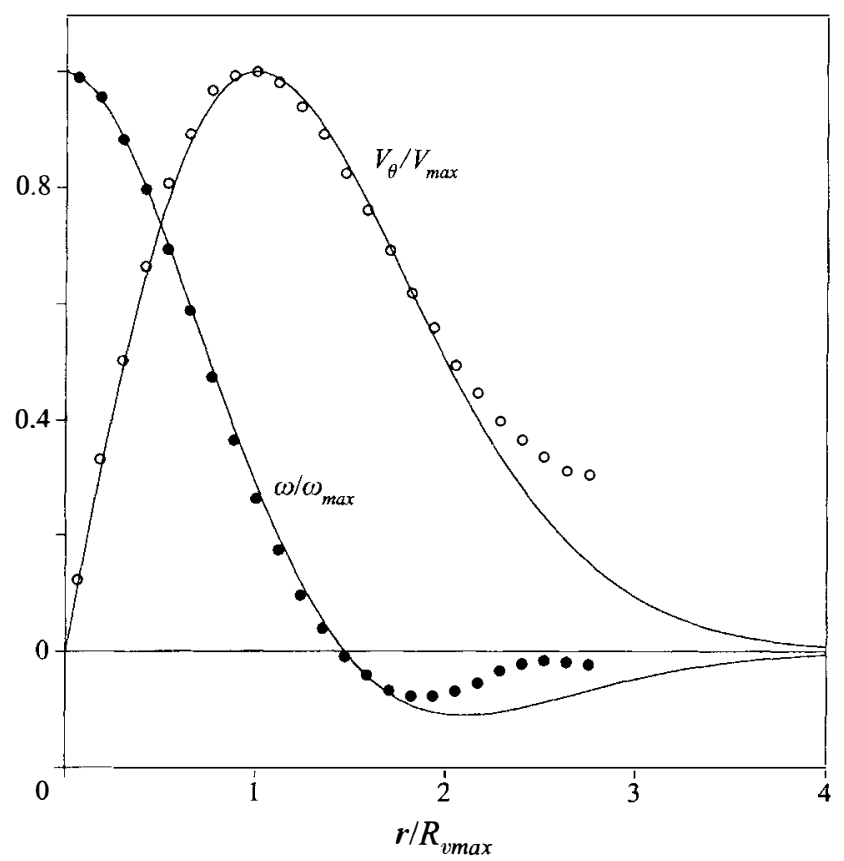

FIGURE 4. Typical radial distributions of the azimuthal velocity (०) and vorticity $(\bullet)$ of a monopolar vortex at $60 \mathrm{~s}$ after forcing. The data are fitted according to equations (3.5) and (3.6) (with $\alpha=1.8$ ) for the velocity and the vorticity profile, respectively, and the fits are represented by the lines; $V_{\max }=0.68 \mathrm{~cm} \mathrm{~s}^{-1}, \omega_{\max }=0.61 \mathrm{~s}^{-1}$ and $R_{v \max }=3.7 \mathrm{~cm}$. This vortex was generated by the tangential-injection method. Experimental parameters: $Q=6 \mathrm{ml} \mathrm{s}^{-1}, \delta t=80 \mathrm{~s}$, linear stratification with $N=2.3 \mathrm{rad} \mathrm{s}^{-1}$.

The Reynolds number $R e$ is defined as $V_{\max } R_{v \max } / v$, with $v$ the viscosity; $\beta$ is the aspect ratio $\sigma / R_{v \max }$, with $\sigma$ a measure of the vertical dimension of the vortex structure. The Reynolds number $R e$ was of $O(100)$, while the aspect ratio $\beta$ was approximately of the order 0.5 ( $\sigma$ was estimated from shadowgraph visualizations shortly after the vortex formation), showing that vertical diffusion of (vertical) vorticity is large compared to horizontal diffusion.

For large $R e$-values, viscous effects are small compared to the advective time scale so that the viscous terms on the right-hand side of (3.4) can to a good approximation be neglected. This results in the vorticity equation for a purely two-dimensional flow. For a stationary structure, equation (3.4) then reduces to $J\left(\omega_{z}, \psi\right)=0$, which implies a functional relationship between $\omega$ and $\psi$. When such a quasi-steady state is chosen as the basic state, the decay of the vortex is described purely by viscous diffusion, as will be discussed in $§ 3.2 .2$. From here on, the flow will be considered as two-dimensional and the index of $\omega_{z}$ will be omitted ( $\omega_{z}$ will be replaced by $\left.\omega\right)$.

Figure 4 shows a typical velocity and vorticity profile of a vortex generated by the tangential injection method in a linearly stratified fluid. Similar profiles were found after forcing by the rotating sphere and the stirrer. The vorticity distribution of the vortex is characterized by a single-signed core enclosed by a ring of oppositely signed vorticity.

A typical sequence of normalized velocity and vorticity profiles of a vortex that was generated by the rotating sphere is shown in figures $5(a)$ and $5(b)$, respectively. The curves reveal a remarkable similarity over time of the vortex core for both profiles. 

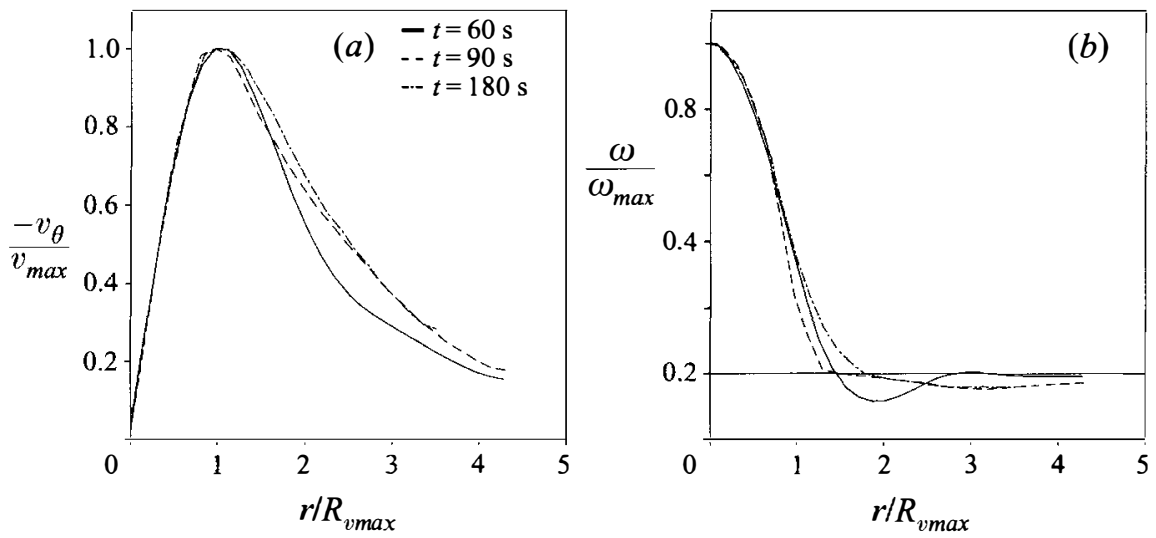

FiguRE 5. The time similarity of the radial distributions of $(a)$ velocity and $(b)$ vorticity. In these graphs the data are represented by lines and the time of each profile is indicated in $(a)$. The monopole was generated by the rotating sphere. Experimental parameters: forcing rotation speed 340 r.p.m., $\delta t=30 \mathrm{~s}$, linear stratification with $N=1.98 \mathrm{rad} \mathrm{s}^{-1}$, sphere diameter $2.5 \mathrm{~cm}$. The maximum values decreased from $V_{\max }=0.58 \mathrm{~cm} \mathrm{~s}^{-1}, \omega_{\max }=0.53 \mathrm{~s}^{-1}$ at $t=60 \mathrm{~s}$ to $0.31 \mathrm{~cm} \mathrm{~s}^{-1}$ and $0.29 \mathrm{~s}^{-1}$ at $t=180 \mathrm{~s}$, respectively.

While the 'tail' of the velocity profile shows a tendency to become less steep, the vorticity profiles show that the outer ring, containing negative vorticity, gradually expands and decreases in amplitude. Simultaneously, the velocity maximum shifts radially outwards, as is shown graphically in figure 6 , where $R_{v \max }$, scaled with its value $R_{0}$ at $t=0$, is plotted versus time. Initially, this radius increases approximately linearly in time (when averaging the oscillations), while after $t \approx 300 \mathrm{~s}$ the expansion rate decreases.

In contrast, the vortices generated by the tangential injection method, which were less perturbed by internal waves, expanded horizontally only a few percent $(\sim 5 \%)$ within $400 \mathrm{~s}$. Also the tail of the vortex did not decrease in steepness as much as the monopolar vortex did. Therefore, the horizontal vortex expansion is believed to be due to the stretching effects induced by internal waves of even modes. When the wave maxima do not coincide with the monopolar vortex centre, such waves lead to an unequal stretching or compression of the vortex structure and thereby perturb its circular shape, which leads to entrainment of ambient fluid. As a consequence the vortex diameter increases. Initially, the internal wave motions are intense and induce considerable entrainment, while with their decay the radial expansion rate of the vortex also decreases (see figure 6) and the vortex size only increases by horizontal diffusion.

With respect to the stability, a useful azimuthal velocity profile of shielded isolated monopolar vortices to compare the data with is given by the velocity profile

$$
v_{\theta}=r \exp \left(\frac{1-r^{\alpha}}{\alpha}\right)
$$

with the corresponding vorticity profile

$$
\omega=2\left(1-\frac{1}{2} r^{\alpha}\right) \exp \left(\frac{1-r^{\alpha}}{\alpha}\right),
$$

where $\alpha$ is a free parameter that controls the steepness of the velocity profile (Carton et al. 1989). Here, the velocity profiles are scaled on the maximum velocity and 


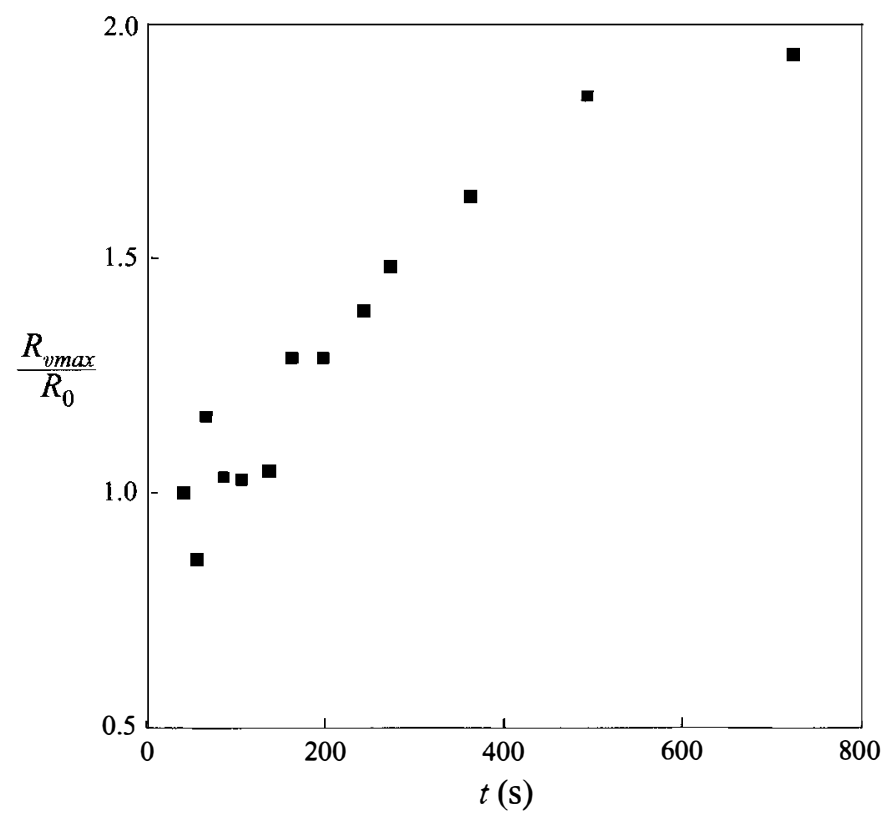

FIGURE 6. The typical growth in radius $R_{v \max }$ scaled with its initial value $R_{0}=2.3 \mathrm{~cm}$. The monopole was generated by the rotating sphere. Experimental parameters: forcing rotation speed 750 r.p.m., $\delta t=5 \mathrm{~s}$, two-layer stratification with $\approx 5 \mathrm{~cm}$ deep interface, in which $N=2.5 \mathrm{rads}^{-1}$, sphere diameter $2.5 \mathrm{~cm}$.

the matching radius (this is at variance with Carton et al. 1989, who scaled on the maximum vorticity). Since the parameter $\alpha$ represents the steepness of the velocity profile, it serves also as a measure of the instability of the vortex due to shear. Carton et al. (1989) found in their numerical study that the monopolar vortices described by (3.5) are unstable for a steepness parameter $\alpha>2$, while they are stable for $\alpha<2$.

For convenience both profiles shown in figure 4 are scaled with their maximum values and the radius $R_{v \max }$ for which the velocity has a maximum. The data for $r<2 R_{v \max }$ are fitted by the least-square method according to the relations (3.5) and (3.6) for the velocity and the vorticity, respectively, and this yields $\alpha \approx 1.6$. Apart from the tails, the theoretical curves for this $\alpha$-value show a good agreement with the experimental observations. For radii $r>2 R_{v \max }$ the vortex flow was not always circularly symmetric, so that the experimental error for a single cross-section could be relatively large for larger $r$-values. Therefore, these data points have been omitted from the fits. In the various experiments that resulted in a stable monopole, the steepness parameter $\alpha$ appeared to vary from $\alpha=1.2$ to $\alpha=1.9$, which is in agreement with the numerical results of Carton et al. (1989) for stable monopolar vortices.

By plotting for each grid point the value of the vorticity against the value of the stream function one obtains a so-called $\omega, \psi$-scatter plot. Figure 7 shows two characteristic $\omega, \psi$-scatter plots of the vortex just after generation (7a) and after many turnover times $(7 b)$. The negative part of the $\omega, \psi$-relation represents the ring of negative vorticity, while the vorticity maximum represents the vortex centre. Note that the stream function in these plots is related to the radial coordinate of the vortex. Although the vortex core appears to be well defined by a linear relation, the negative ring shows considerable scatter (see figure $7 a$ ), which is most likely due to entrainment of ambient fluid. This ring continues to expand until a stationary 

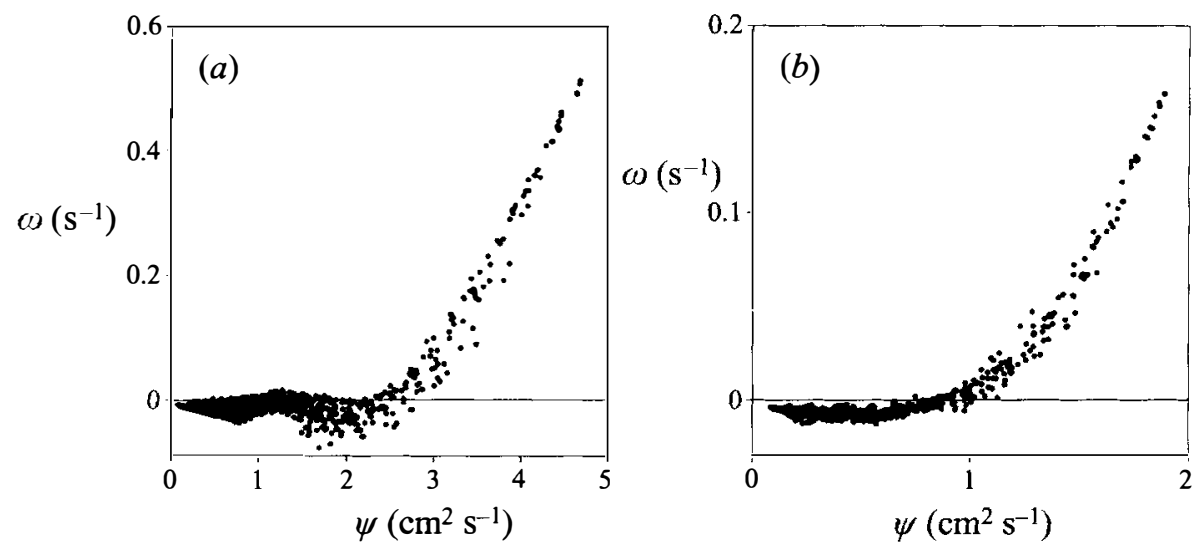

FIGURE 7. Typical evolution in the $\omega, \psi$-relation of the monopolar vortex, at $t=60 \mathrm{~s}(\boldsymbol{a})$ and at $t=290 \mathrm{~s}(b)$. Experimental parameters: see caption of figure 5.

vortex has been formed, apparently, with a very wide region of weak negative vorticity around the core, as visible in figure $7(b)$. The $\omega, \psi$-relation for the vortex core remains approximately linear.

\subsection{Comparison with decay models}

\subsubsection{Comparison with the Bessel-vortex model}

In view of the $\omega, \psi$-relations discussed above the vortex-core region should be modelled with a linear relationship $\omega=k^{2} \psi$, where $k$ is a constant. With $\omega=-\nabla^{2} \psi$, one obtains, after introducing cylindrical coordinates and assuming axisymmetry

$$
\frac{\mathrm{d}^{2} \psi}{\mathrm{d} r^{2}}+\frac{1}{r} \frac{\mathrm{d} \psi}{\mathrm{d} r}=-k^{2} \psi
$$

which is the Bessel equation of zero order. Allowing only the physically realistic solutions we obtain for the stream function, vorticity and the azimuthal velocity, respectively,

$$
\begin{aligned}
\psi(r) & =\frac{A}{k^{2}} J_{0}(k r), \\
\omega(r) & =\frac{1}{r} \frac{\mathrm{d}}{\mathrm{d} r}\left(r v_{\theta}\right)=A J_{0}(k r), \\
v_{\theta}(r) & =-\frac{\mathrm{d} \psi}{\mathrm{d} r}=\frac{A}{k} J_{1}(k r),
\end{aligned}
$$

with $J_{0}$ and $J_{1}$ the zeroth- and first-order Bessel functions of the first kind, and $A$ a constant. Because we model the core vortex only, the solution is truncated at the first zero of $J_{0}$, implying a single-signed vorticity distribution. The first zero of $J_{0}$ yields the dispersion relation $k R=2.40 \ldots$, where $R$ is the radius of the vorticity-containing region. An equivalent relation can be derived for the radius of maximum velocity $R_{v \max }$, which yields

$$
k R_{v \max }=1.84 \ldots .
$$

At radius $R$ the rotational flow is matched with an external potential flow given by

$$
v_{\theta}(r)=-\frac{A}{k r} J_{1}(k R) R, \quad r>R .
$$




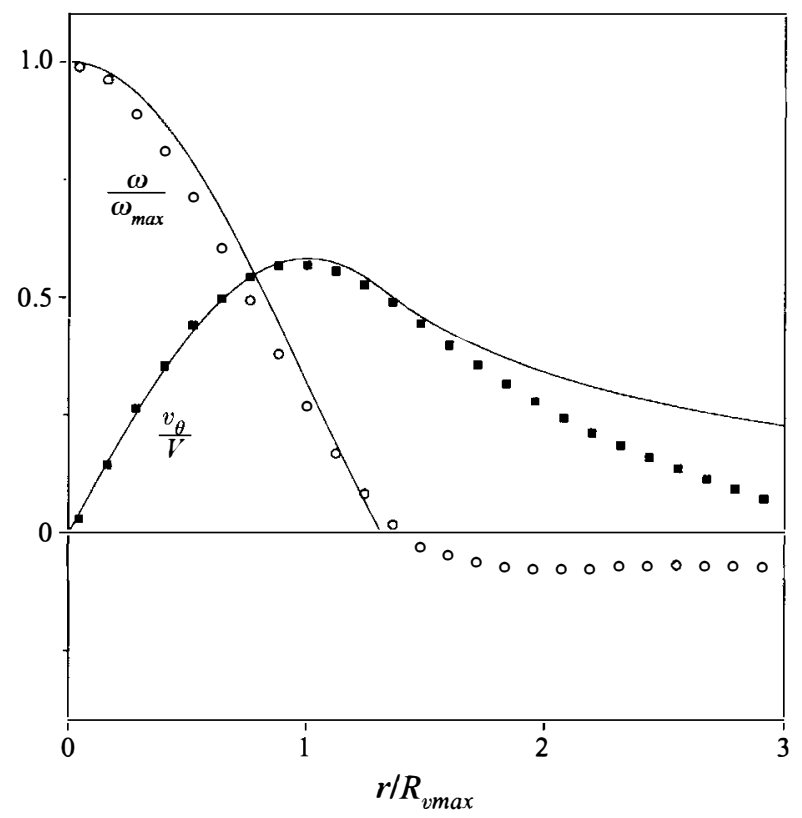

FIGURE 8. Velocity and vorticity data for a monopolar vortex compared with the Bessel-type vortex represented by the line; the data are represented by symbols. The vorticity is scaled with the maximum vorticity value $\omega_{\max }=0.227 \mathrm{~s}^{-1}$ and the velocity with $V=\omega_{\max } / k=0.37 \mathrm{~cm} \mathrm{~s}^{-1}$ where $k=1.841 \ldots / R_{v \max }$ according to equation (3.9) (the maximum velocity was $0.21 \mathrm{~cm} \mathrm{~s}^{-1}$ ). The radial coordinate is scaled with $R_{v \max }=3.0 \mathrm{~cm}$. The monopolar vortex was generated by a rotating sphere. Experimental parameters: see caption of figure 6.

In the rest of this paper we will refer to this model as the Bessel vortex. A similar solution for a non-isolated vortex was derived by Leith (1984). Leith assumed a minimum enstrophy state, based on the idea that in slightly viscous two-dimensional flows the enstrophy dissipates much faster than the energy, and he derived models for both an isolated and a non-isolated vortex. Although it is questionable if the present viscously dissipating flows have enough energy to ever reach such a minimum-enstrophy state, the measurements showed reasonable agreement with both Leith-vortex models (1984). In the context of the present paper we show only the comparison with the Bessel vortex that is based simply on a linear $\omega, \psi$-relationship.

Figure 8 shows the (scaled) vorticity and velocity profiles of the Bessel vortex and the measured profiles. Because in the present experiments the vorticity in the negative ring of the vortex is relatively low and the $\omega, \psi$-relation in the core is nearly linear, a reasonable agreement is obtained with the Bessel-vortex model. Since the radius $R_{v \max }$ can be measured from velocity profiles, the relation (3.9) can be compared with the experiments. The $\omega, \psi$-relation was least-square fitted for $\omega>0$ and this yielded a value for $k^{2}$, while the value of $R_{v \max }$ was determined from cross-sectional velocity profiles. This resulted in an experimental value $k R_{\text {max }}=1.79 \pm 0.06$, which corresponds well with the model value of $1.84 \ldots$. These results show that the laboratory vortices are reasonably well described by this Bessel-vortex model.

\subsubsection{The decay models}

In previous related studies (see Flór \& van Heijst 1994; Flór, van Heijst \& Delfos 1995 ) it has been shown that the decay of planar vortex structures in stratified fluids is mainly governed by vertical diffusion of vertical vorticity. Following the same 
approach as in Flor et al. (1995), it is assumed that the motion is confined in a thin disk-shaped region, where the vortex lines are directed vertically and the vortex tilting due to the shear is negligible. Then, for circularly symmetric flow structures the evolution of the vorticity is described by

$$
\frac{\partial \omega}{\partial t}=v \nabla_{h}^{2} \omega+v \frac{\partial^{2} \omega}{\partial z^{2}},
$$

where the decay of the horizontal flow is mainly governed by (vertical) diffusion of vertical vorticity as was shown by the scaling in $\S 3.1$.

For the flow around the mid-level $z=0$ where the shear $\partial v_{\theta} / \partial z=0$, and the vorticity is directed vertically, we present three approximate models in order of increasing sophistication. The basic horizontal flow is approximated by the Besseltype vortex (3.8), which, because of its linear $\omega, \psi$-relation, allows one to develop a simple process model which can readily be compared with the data.

\section{(a) Purely two-dimensional decay}

As a first approximation the decay of the planar vortex flow can be considered as purely two-dimensional, by neglecting the last term in (3.10). For the case of a circular vortex with $\omega=k^{2} \psi$, discussed in the previous section, one obtains (see also Batchelor 1967, p. 537)

with the decaying vortex solution

$$
\frac{\partial \omega}{\partial t}=v \nabla^{2} \omega=-v k^{2} \omega
$$

$$
\omega(r, t)=\omega_{o}(r) \exp \left(-t / \tau_{2 D}\right)
$$

where $\omega_{o}(r)$ is the vorticity according to the model vortex $(3.8 b)$ and

$$
\tau_{2 D}=\left(v k^{2}\right)^{-1}
$$

the two-dimensional decay time.

(b) The 'constant-thickness' model

A somewhat more refined model can be constructed by taking into account the vertical structure of the planar vortex. The vertical distribution of the horizontal velocity field can be closely approximated by a Gaussian profile of the form $\exp \left(-z^{2} / 2 \sigma^{2}\right)$, with $\sigma$ a vertical scale (see Flór et al. 1995). Assuming that the structure has a constant thickness $2 \sigma$ during (at least) the first stages of the decay, the vorticity distribution in the region $z \ll \sigma$ can be approximated by

$$
\omega(r, z, t)=\omega_{o}(r) \exp \left(-z^{2} / 2 \sigma^{2}\right) h(t),
$$

where $h(t)$ is a time-dependent amplitude function. Substition of (3.14) into (3.10) yields

$$
\frac{\partial \omega}{\partial t}=v\left(-\lambda^{2}+\frac{\epsilon^{2}}{\sigma^{2}}\right) \omega
$$

with $\epsilon=z / \sigma$ and $\lambda^{2}=k^{2}+1 / \sigma^{2}$. Since $z \ll \sigma$, then $|\epsilon| \ll\left(1+\sigma^{2} k^{2}\right)^{1 / 2}$, so that one obtains the approximate solution

$$
\omega(r, z, t)=\omega_{o}(r) \exp \left(-t / \tau_{c t}\right) \exp \left(-z^{2} / 2 \sigma^{2}\right)-O\left(v \epsilon^{2} t / \sigma^{2}\right)
$$

with

$$
\tau_{c t}=\left(v \lambda^{2}\right)^{-1}
$$


The last term in (3.16) is negligible as $t \ll \sigma^{2} / v \epsilon^{2}$, which is easily met in most experiments as long as $\epsilon$ is small. Apparently, the decay is again exponential, but due to the thickness value the decay time will be shorter.

\section{(c) The vertical diffusion model}

A more accurate model is obtained when the vertical diffusion of the (vertical) vorticity is allowed to result in an increasing thickness of the vortical region. The model is again restricted to the thin region around the mid-plane level $z=0$ where the vorticity is directed vertically, for which we put

$$
\omega(r, z, t)=\omega_{o}(r) \gamma(z, t)
$$

with $\omega_{o}(r)$ again the Bessel-vortex solution (3.8), and $\gamma(z, t)$ an amplitude function. Substitution into (3.10) yields

$$
\frac{\partial \gamma}{\partial t}=-v k^{2} \gamma+v \frac{\partial^{2} \gamma}{\partial z^{2}}
$$

which, after substitution of $\gamma=\Phi(z, t) \exp \left(-v k^{2} t\right)$, leads to a diffusion equation for $\Phi$ :

$$
\frac{\partial \Phi}{\partial t}=v \frac{\partial^{2} \Phi}{\partial z^{2}} \text {. }
$$

Assuming that initially the vorticity is confined to a thin region at mid-plane depth, according to $\Phi(t=0)=\Phi_{o} \delta(z)$, the solution of (3.20) is

$$
\Phi(z, t)=\frac{\Phi_{o}}{t^{1 / 2}} \exp \left(-z^{2} / 4 v t\right)
$$

Although the flow has a certain thickness at $t=0$ and strictly is not described by a thin $\delta$-like initial condition, the structure expands vertically during its decay so that the initial thickness will be relatively small. One may expect, therefore, that this solution still will give a reasonable description of the flow evolution. The solution for the vorticity is then

$$
\omega(r, z, t)=\frac{\omega_{o}(r)}{t^{1 / 2}} \exp \left(-t / \tau_{d i f}\right) \exp \left(-z^{2} / 4 v t\right),
$$

with the time scale

$$
\tau_{d i f}=\left(v k^{2}\right)^{-1},
$$

and the constant $\Phi_{o}$ being incorporated in the amplitude of $\omega_{o}(r)$. Note that this time scale $\tau_{d i f}$ is identical to $\tau_{2 D}$, see (3.13), as derived for the purely two-dimensional decay. However, owing to the extra factor $1 / t^{1 / 2}$ the decay in $\omega$ will be faster in this case than for the purely two-dimensional case.

\subsubsection{Comparison with experimental results}

\section{(a) The constant-thickness model}

The most appropriate quantity to characterize the decay of the vortex is its maximum vorticity $\omega_{\max }$, which decays due to viscous diffusion. Related quantities that can be determined are $V_{\max } / R_{v \max }$ and $\Gamma / R^{2}$ with $\Gamma$ the circulation and $R$ the vortex radius, where $R$ is determined from scatter plots with the relation $R=2.401 / k$, and the circulation $\Gamma$ was obtained by integrating numerically over the interpolated grid according to $\Gamma=\frac{1}{2} \Sigma_{i, j}\left|\omega_{i, j}\right| \Delta x \Delta y$, where $\Delta x \Delta y$ is the surface of one mesh of the grid. 

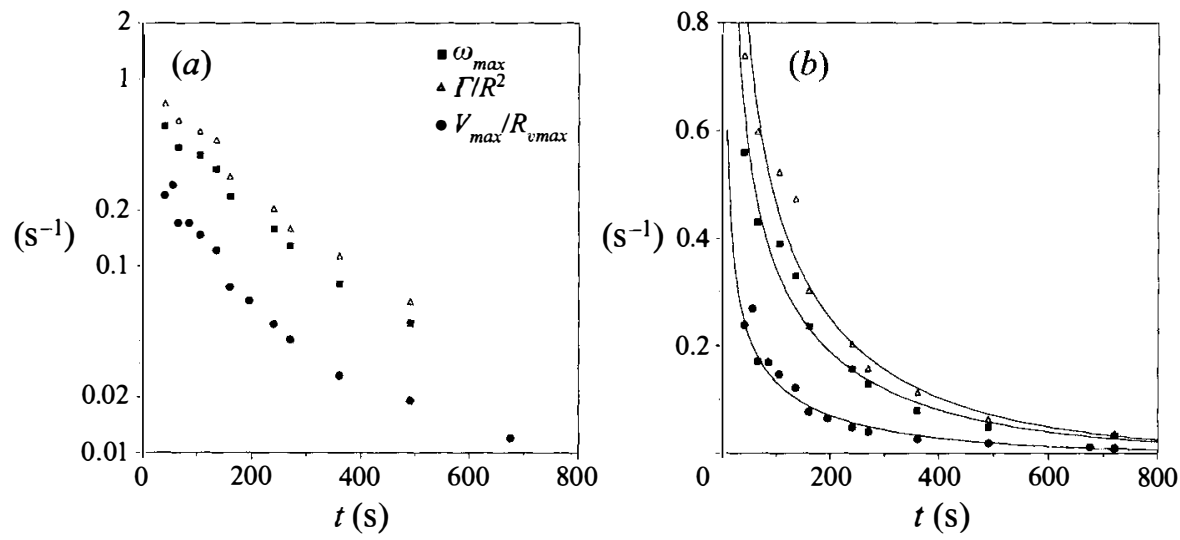

FIGURE 9. The decay in vorticity $\omega_{\max }, V_{\max } / R_{\text {vmax }}$ and $\Gamma / R^{2}$ as a function of time plotted $(a)$ in a log-linear plot displaying the exponential decay and $(b)$ fitted with the relation $t^{-1 / 2} \exp \left(-t / \tau_{d i f}\right)$ according to the diffusion model (3.22). Experimental parameters: see text.

$$
\begin{array}{ccccc}
\text { exp. } & \tau_{c t}\left(\omega_{\max }\right) & \tau_{c t}\left(V_{\max } / R_{v \max }\right) & \tau_{c t}\left(\Gamma / R^{2}\right) & \tau_{c t}^{\prime} \\
& (\mathrm{s}) & (\mathrm{s}) & (\mathrm{s}) & (14) \\
\text { I } & 187 \pm 10 & 156 \pm 14 & 189 \pm 15 & 237 \pm 64 \\
\text { II } & 240 \pm 20 & 213 \pm 19 & 216 \pm 17 & 235 \pm 88
\end{array}
$$

TABLE 1. Decay times for the constant-thickness model.

In order to measure the decay of a monopolar vortex, the streak pictures of two typical experiments were digitized. In both experiments (I and II) the flow was generated by a rotating sphere of diameter $2.5 \mathrm{~cm}$. In experiment I the sphere rotated for approximately $15 \mathrm{~s}$ with a rotation speed of $\Omega=344$ r.p.m. in a linearly stratified fluid with buoyancy frequency $N=2.0 \mathrm{rad} \mathrm{s}^{-1}$. Six streak pictures taken between $0 \mathrm{~s}$ and $290 \mathrm{~s}$ were digitized. In experiment II the flow was forced for 5 seconds, with $\Omega=750$ r.p.m. and $N=2.5 \mathrm{rad} \mathrm{s}^{-1}$. The experiment lasted $720 \mathrm{~s}$ and 10 streak pictures were digitized.

Figure $9(a)$ shows the behaviour of $\omega_{\max }, V_{\max } / R_{v \max }$, and $\Gamma / R^{2}$ as a function of time for experiment II. In this graph the vertical axis is taken logarithmic so that an exponential decay is displayed by a straight line; the plot clearly displays an approximately similar behaviour for all three quantities. By taking least-square fits with an exponential curve the decay time for each quantity was determined; the values for the two different experiments are shown in table 1.

In order to compare these values with an independently determined 'theoretical' decay value $\tau_{c t}^{\prime}=\left(v k^{2}+v / \sigma^{2}\right)^{-1}$, in a similar experiment the vortex thickness $\sigma$ was estimated from shadowgraph visualizations. Vorticity diffuses relatively slowly, so that one may assume that the density structure in the shadowgraph visualization just after forcing also represents the initial thickness of the (vorticity) structure. From these visualizations a mean value $\sigma=3.0 \pm 0.6 \mathrm{~cm}$ was measured. Least-square linear fits of the $\omega, \psi$-scatter plots for $\omega>0$ provided average values $k_{I}^{2}=0.31 \pm 0.05 \mathrm{~cm}^{-2}$ for experiment I, and $k_{I I}^{2}=0.36 \pm 0.13 \mathrm{~cm}^{-2}$ for experiment II, yielding the 'theoretical' decay values $\tau_{c t}^{\prime}$.

Although the estimation of the vortex thickness is rough, the decay values $\tau_{c t}^{\prime}$ 


$\begin{array}{ccccc}\text { Exp. } & \tau_{\text {dif }}\left(\omega_{\max }\right) & \tau_{\text {dif }}\left(V_{\max } / R_{v \max }\right) & \tau_{\text {dif }}\left(\Gamma / R^{2}\right) & \tau_{d i f}\left(1 / v k^{2}\right) \\ & (\mathrm{s}) & (\mathrm{s}) & (\mathrm{s}) & (\mathrm{s}) \\ \text { I } & 405 \pm 69 & 282 \pm 31 & 407 \pm 69 & 322 \pm 60 \\ \text { II } & 403 \pm 36 & 374 \pm 30 & 375 \pm 30 & 319 \pm 72\end{array}$

TABLE 2. Decay times for the vertical diffusion model.

approach the experimental decay values $\tau_{c t}$ reasonably well. However, on large time scales the approximation of a constant thickness is not valid anymore and larger deviations from the model occur (see figure $9 a$ ).

\section{(b) the vertical diffusion model}

In order to compare the decay values with the vertical diffusion model, the data for $\omega_{\max }, V_{\max } / R_{v \max }$ and $\Gamma / R^{2}$ were least-square fitted according to equation (3.22), as is shown in figure $9(b)$. The time at which the forcing was stopped was taken as $t=0$. The graph clearly shows that the model describes a similar decay trend as the experiments. The decay time scales obtained are shown in table 2, where the decay values $\tau_{\text {dif }}=1 / v k^{2}$ were determined with the values $k_{I}^{2}$ and $k_{I I}^{2}$ obtained from scatter plots, as mentioned above. Generally, the experimental values are higher than the 'theoretical' decay value $\tau_{d i f}=1 / v k^{2}$. Presumably, this is caused by the assumption of a $\delta$-shaped initial condition in the model: in reality a vortex structure with a finite thickness at $t=0$ diffuses slower than a thin $\delta$-shaped vorticity distribution. Nevertheless, the three different experimental decay values are for both experiments - within the experimental error - in agreement with the 'theoretical' decay value $\tau_{d i f}\left(1 / v k^{2}\right)$, thus showing that the model gives a reasonable description of the decay. Although both decay models appear to be of use to predict the decay of monopolar structures, the diffusion model shows better agreement with the data on larger time scales (see figure 9).

\section{Unstable monopolar vortices}

Although in most experiments the forcing resulted in a stable monopolar vortex, in some experiments instabilities grew and led to the formation of a multipolar vortex. Unstable monopolar vortices were observed for the forcing by the stirrer or by the tangential-injection method, while the vortices generated by the rotating sphere were generally stable.

The vortices generated by the stirrer were the most unstable ones, probably due to the large-amplitude perturbations that resulted from the rigorous mixing during the forcing. The vortices thus created almost instantly transformed into a tripolar vortex so that the preceding monopolar vortex was hardly perceivable. The vortices created by the tangential-injection method were less perturbed and a gradual transition into a higher-mode vortex could be observed.

The tripoles were observed to transform either to a monopolar vortex as a consequence of the faster decay of the satellite vortices, or to split up into two dipolar vortices. The cases where the vortex split up into two dipoles were very similar to the flow evolution of anticyclonic (two-layer) vortices in rotating fluids (see Griffiths \& Linden 1981) and in homogeneously rotating flows (see Kloosterziel \& van Heijst 1991) and are not further discussed in this paper.

As mentioned in $\$ 3.1$, the monopolar vortices can be barotropically unstable due 


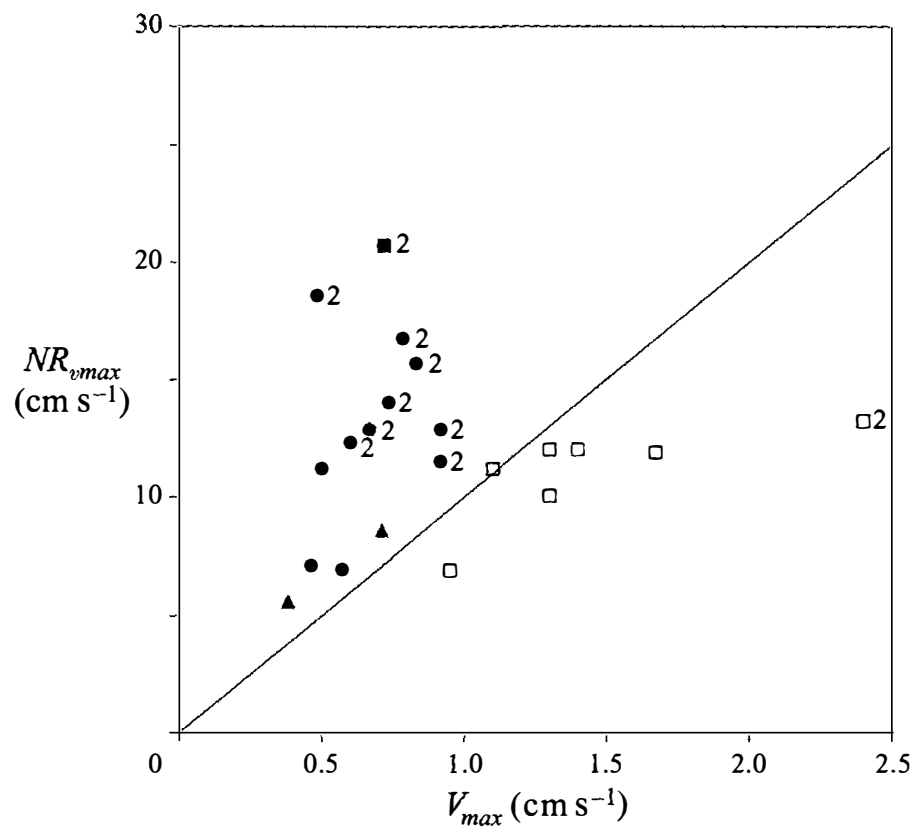

FIGURE 10. Stability diagram, showing experimental observations of monopolar vortices as a function of the parameters $V_{\max }$ and $N R_{v \max }$, with $R_{v \max }$ the radius at which the maximum velocity magnitude $V_{\max }$ was measured shortly $(\approx 20 \mathrm{~s})$ af ter the monopole generation. Experiments that resulted in unstable vortices are denoted by open symbols, whereas stable vortices are denoted by filled symbols. The square, circular and triangular symbols refer to the different forcings, i.e. stirrer, rotating sphere and tangential injection method, respectively. The number 2 indicates that the experiment was performed in the thick interface of a two-layer stratification. The solid line corresponds with $F=0.1$, and marks the boundary between the stable and unstable regimes.

to shear instability, when the steepness parameter for the velocity profile $\alpha>2$. Since stable monopolar vortices have been found only for $\alpha<2$ one may expect that, in agreement with the numerical results of Carton et al. (1989), the present monopoles are also unstable for $\alpha>2$. However, since the scatter in the data for $r>R_{v \max }$ was too large during the initial vortex formation, no precise value of $\alpha$ could be measured.

In order to quantify the influence of baroclinic effects, a Froude number of the vortex was defined as $F=V_{\max } / N R_{v \max }$ with $V_{\max }$ the maximum velocity and $R_{v \max }$ the corresponding radius just after the vortex generation. As follows from equation (3.3), this Froude number represents a measure of the slope $\left(h / R_{v \max }\right)$ of the isopycnals in the vortex. It appeared that the flow was unstable for $F \geqslant 0.1$ and vortex structures with a higher mode formed, while for $F<0.1$ the monopolar vortices were found to be stable (see figure 10). Steep slopes in the isopycnals also imply a large aspect ratio between vertical and horizontal dimensions of the vortex, which suggests that the instability is due to vortex tilting. For small Froude numbers $(F<0.1)$ the buoyancy force dominates over the inertia forces, so that baroclinic instabilities are expected to be suppressed.

In the next subsections, attention is focused on the formation of the tripolar vortex and the formation of a persistent triangular vortex. Because tripolar and triangular vortex structures have not been observed in a stratified fluid before, their characteristic properties will be studied in detail in $\S 5$. 

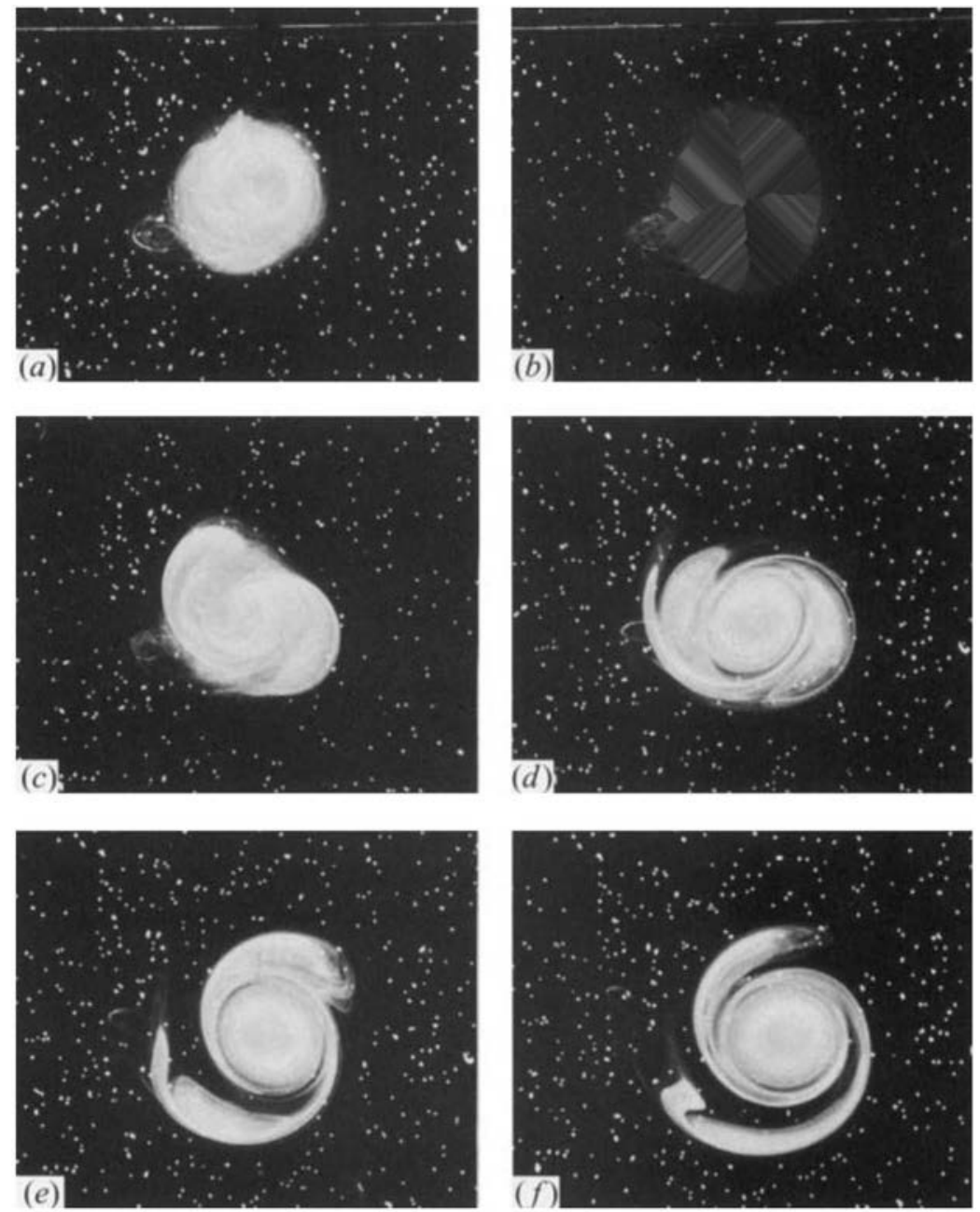

FIGURE 11. A sequence of plan-view photographs showing the evolution of the dye distribution of an unstable monopolar vortex, that led to the formation of a tripolar vortex and subsequently, due to viscous effects, to a monopolar vortex again. The monopole was generated by the tangential-injection method with a cylinder of diameter $12 \mathrm{~cm}$ and height $3 \mathrm{~cm}$, with the dye initially distributed within the cylinder; the rotation sense of the vortex is anticlockwise. The photographs were taken at $t=45 \mathrm{~s}(a) ; 55 \mathrm{~s}(b) ; 80 \mathrm{~s}(c) ; 240 \mathrm{~s}(d) ; 440 \mathrm{~s}(e)$ and $660 \mathrm{~s}(f)$. Experimental parameters: $Q=1 \mathrm{ml} \mathrm{s}^{-1}$, linear stratification with $N=2.0 \mathrm{rad} \mathrm{s}^{-1}, \delta t=24 \mathrm{~s}$. Each frame shows an area of $49.5 \times 34.5 \mathrm{~cm}$.

\section{Qualitative observations}

Figure 11 shows a typical dye visualization of the evolution of an unstable monopolar vortex that was generated by the tangential-injection method. The dye was released in the cylinder before it was lifted out and displays approximately the initial vortical flow. Almost instantly after the generation of this monopolar vortex (figure $11 \mathrm{a}$ ) mode- 2 perturbations appeared to grow and the vorticity pattern reorganized into a r.:ntral slightly elongated vortex with two counter-rotating satellite vortices; this newly formed configuration rotated as a compact structure in an anticlockwise direction around its central axis (see figure $11 b-d$ ). By entrainment of ambient fluid the structure expanded horizontally; the distance between the satellites and the core 


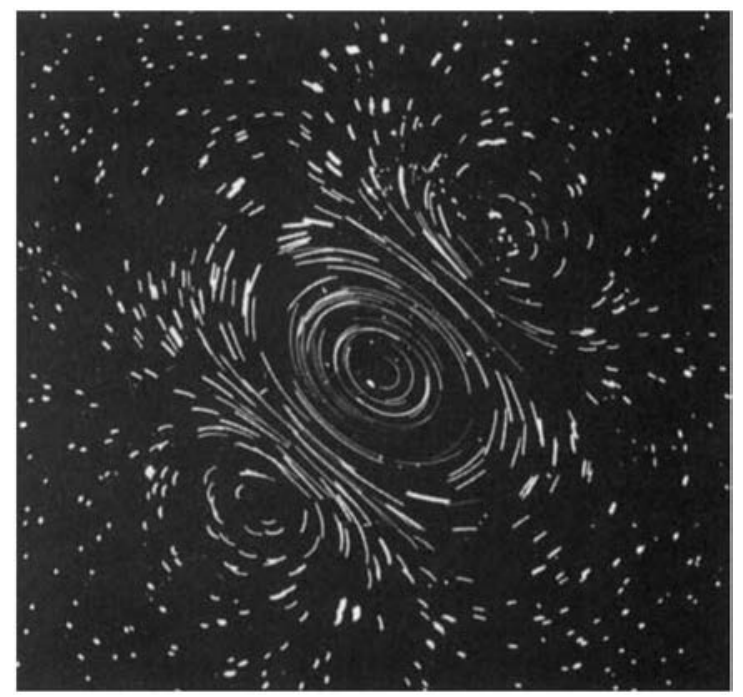

FIGURE 12. Streak photograph of a stable tripolar vortex, generated by the stirrer in a linearly stratified fluid. The exposure time was $6 \mathrm{~s}$. The frame shows an area of $28 \times 29 \mathrm{~cm}$. Experimental parameters: forcing rotation rate 200 r.p.m., $\delta t=10 \mathrm{~s}$, linear stratification with $N=2.8 \mathrm{rad} \mathrm{s}^{-1}$.

vortex increased while the strength of the satellites decreased, resulting in a slower rotation speed (see figure $11 c-e$ ). Probably due to viscous effects, eventually the weakened satellites were torn into vortex filaments by the shear induced by the core vortex, while the core vortex expanded horizontally and obtained a more or less circular shape. Finally, the remains of the secondary vortices were wrapped around the core vortex, gradually resulting in a monopolar vortex (see figure $11 e, f$ ).

Although this tripole appeared to be unstable, its formation is similar to that observed in a rotating fluid by Kloosterziel \& van Heijst (1991), van Heijst et al. (1991) and in numerical simulations by Carton et al. (1989) and Orlandi \& van Heijst (1992). The present tripole, however, had weak satellites and a core that is less elongated than in their observations. This is probably due to a different steepness of the velocity profile of the initial monopole (here probably $\alpha \approx 2$ ). Similar tripolar vortices with weak satellites were also found by Orlandi \& van Heijst (1992) in a two-dimensional numerical simulation of a monopolar vortex described by equation (3.5) with $\alpha=2$. In the rotating fluid experiments on tripoles by van Heijst et al. (1991) the strength of initially weak satellites increased by the advection of vorticity (due to the secondary circulation driven in the Ekman layer at the bottom boundary), and the satellites became more pronounced during the flow evolution.

The tripolar vortices that were generated by the stirrer appeared to have strong side vortices and showed much more similarity with those observed in the numerical simulations of Carton et al. (1989) and the laboratory observations of Kloosterziel \& van Heijst (1991). An example of such a tripole is presented in figure 12; this tripole was symmetric and appeared to persist for a longer time.

However, most tripolar vortices appeared to be asymmetric. Internal waves of even modes - with a length of at least a vortex diameter - alternatingly stretch or compress the vortex region. Such waves may cause an asymmetric stretching (compression) of the core vortex and the satellites when the wave maximum does not coincide with the centre of the core-vortex. This results in a difference in the separation distances between the core vortex and its satellites. 

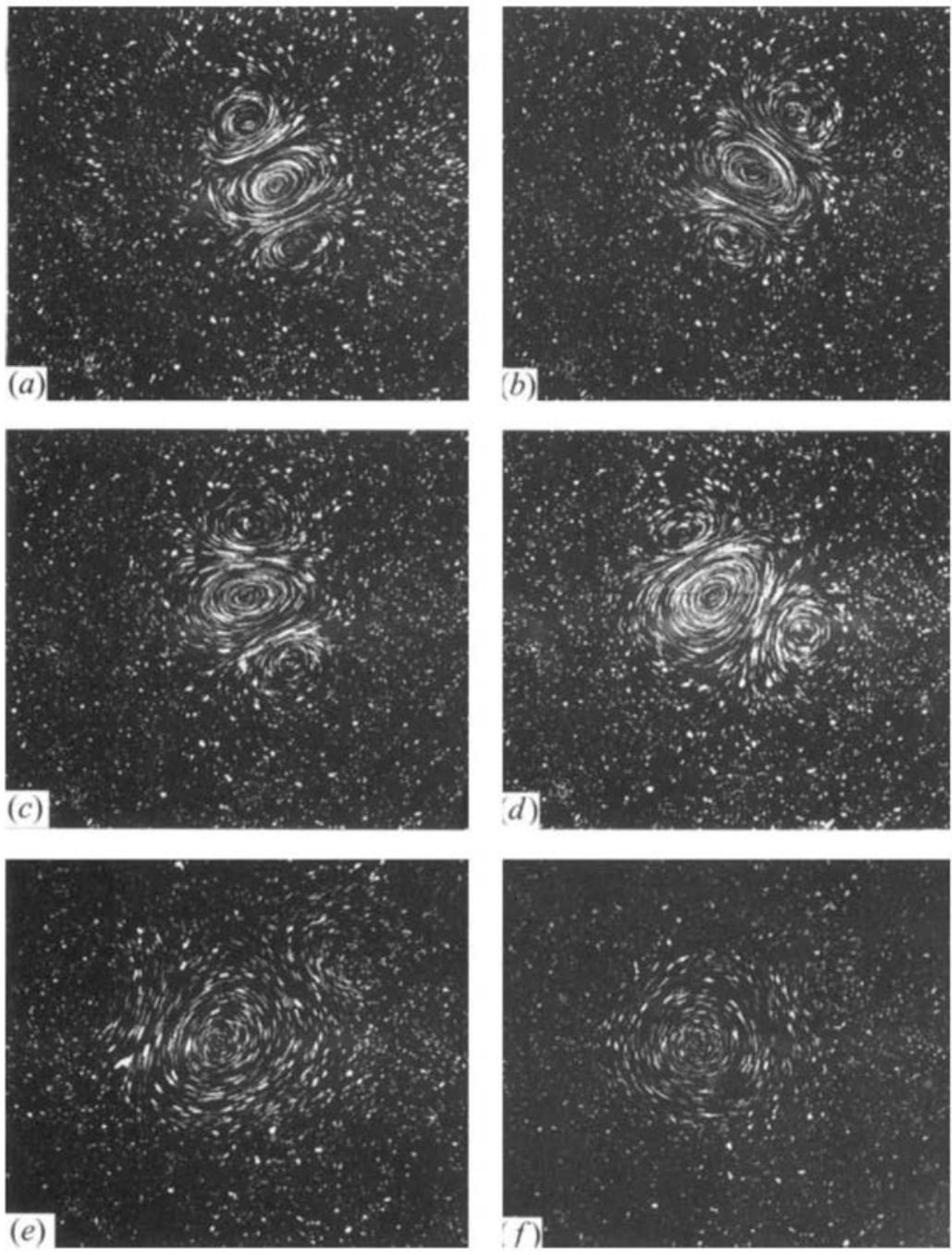

FigURE 13. A sequence of streak photographs showing the evolution of an unstable tripolar vortex (generated by the stirrer) that eventually led to a triangular-like vortex. The photographs were taken at $t=10 \mathrm{~s}(a) ; 33 \mathrm{~s}(b) ; 44 \mathrm{~s}(c) ; 56 \mathrm{~s}(d) ; 145 \mathrm{~s}(e) ; 250 \mathrm{~s}(f)$. Each frame shows an area of $70 \times 53 \mathrm{~cm}$. Experimental parameters: forcing rotation rate $\approx 200$ r.p.m., $\delta t=5$ s, two-layer stratification with $N=4.4 \mathrm{rad} \mathrm{s}^{-1}$.

The asymmetric tripoles appeared to be unstable, eventually, and a monopolar vortex was observed to form when the side vortices were weak, as was the case for the vortices generated by the tangential-injection method shown in figure 11 . When the side vortices were relatively strong, as in the case of a tripole resulting from forcing with the stirrer, the vortex either split up into dipoles or formed higher-mode structures, as will be discussed below.

Figure 13 shows the particle streak visualization of the typical evolution of an unstable tripolar vortex generated with the stirrer. Initially, the two separation distances between the satellite centres and the core centre are slightly different and the three vortex centres are misaligned (see figure 13a,b). This tripolar structure rotates in anticlockwise direction. During the flow evolution, the core vortex expands and gradually changes in shape from oval to triangular, while the angle between 

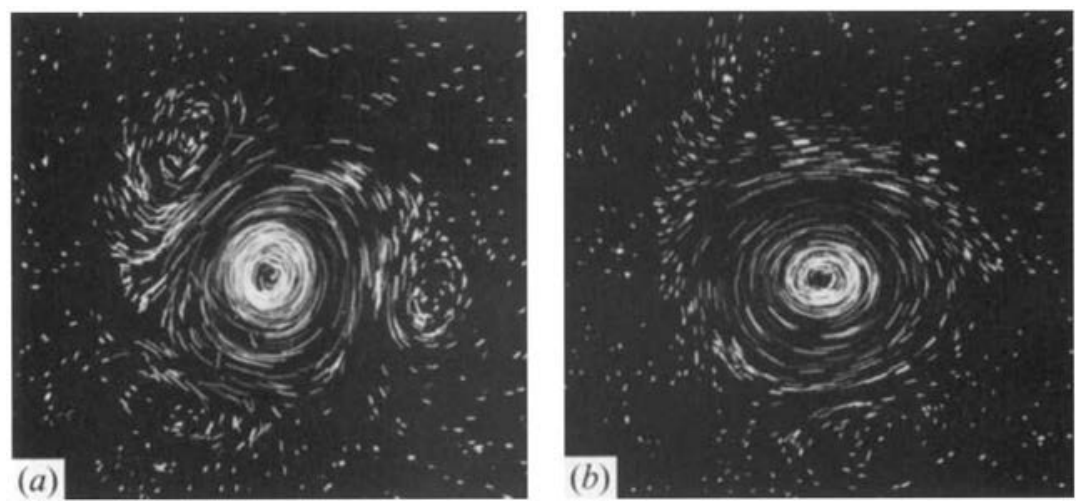

FIGURE 14. Two streak photographs, showing an unstable triangular vortex $(a)$, as generated by the tangential-injection method, that led to the formation of a weak tripolar vortex $(b)$. The pictures are taken at $(a) t=50 \mathrm{~s}$; and $(b) 100 \mathrm{~s}$, with exposure times $2 \mathrm{~s}$ and $4 \mathrm{~s}$, respectively. Experimental parameters: $Q=3.8 \mathrm{ml} \mathrm{s}^{-1}, \delta t=21 \mathrm{~s}$, linear stratification with $N=2.1 \mathrm{rad} \mathrm{s}^{-1}$, cylinder diameter $12 \mathrm{~cm}$. Each frame represents an area of $30 \times 30 \mathrm{~cm}$.

the three vortex centres increases slightly (see figure $13 c, d$ ). Eventually, the vortex structure obtained a triangular shape, that persisted during the further decay (figure $13 e, f)$. Although particle streaks of this vortex are relatively short and not all the secondary vortices are clearly visible (because of a lack of particles in some areas), vorticity contour pictures shown in the next section indicate the presence of a triangular core vortex accompanied by three satellite vortices at its sides.

In a number of experiments, mode-3 perturbations were also observed to grow immediately after the forcing was stopped, and resulted in the formation of a triangular vortex with three satellite vortices, as is clearly demonstrated in figure $14(a)$. However, these vortex structures were unstable and transformed into either a weak tripole (see figure $14 \mathrm{~b}$ ) or a monopolar vortex, similar to the laboratory observations of triangular vortices by Kloosterziel \& van Heijst (1991) and Carnevale \& Kloosterziel (1994).

Triangular vortices have been also observed in two-dimensional numerical simulations by Carton (1992) and Carnevale \& Kloosterziel (1994). The latter authors suggested that the triangular vortex is unstable for small perturbations with an amplitude above a small threshold value. This was demonstrated by Morel \& Carton (1994) who perturbed the tripole with a mode- 3 strain field. The triangular vortices were found to be unstable above a perturbation amplitude of $1 \%$ of the vorticity amplitude. In the present experiments, the triangular vortex structures that emerged almost instantly after forcing, i.e. when perturbations are still large, were unstable, while they appeared to be more persistent at later stages.

\section{Multipolar vortices: measurements}

\subsection{The tripole}

Typical contour plots of the vorticity and stream function of the symmetric tripolar vortex are shown in figures $15(a)$ and $15(b)$, respectively. These plots clearly reveal a tripolar vortex consisting of a core vortex with two secondary vortices of the other sign and a similar distribution in the stream function relative to the co-rotating frame after correction for rotation. The vorticity profile along a cross-section through the 

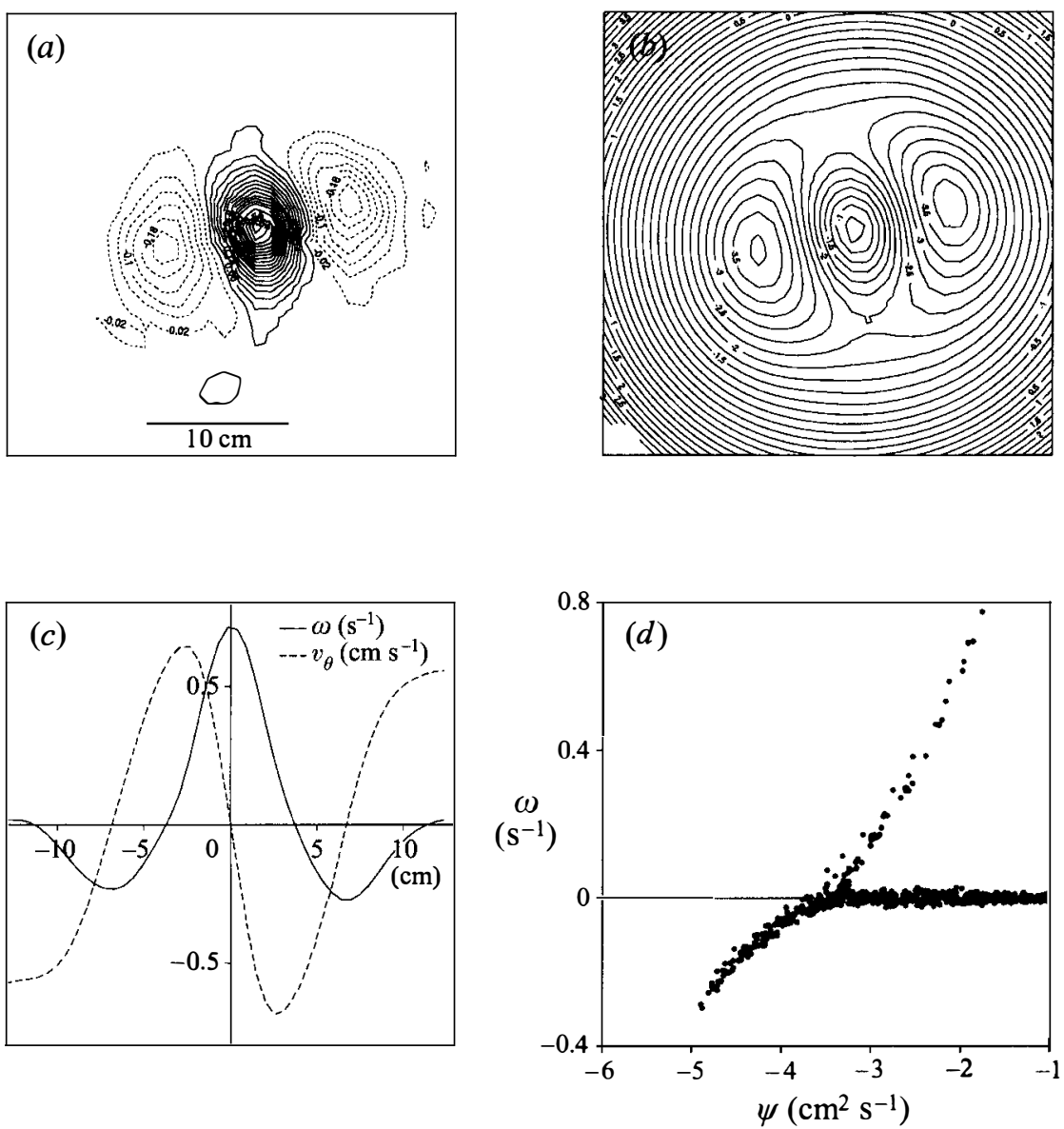

FIGURE 15 . Isoline plots of the vorticity $(a)$ and stream function $(b)$ relative to the co-rotating frame of reference of the tripolar vortex; $(c)$ characteristic profile of the azimuthal velocity (dashed line) and the vorticity profile (solid line) along a line through the three vortex centres; $(d)$ the $\omega, w$-scatter plot. Experimental parameters: see figure 12.

vortex centres of this tripole is shown in figure $15(c)$ and reveals clearly that this tripole has a continuous vorticity distribution.

The corresponding $\omega, \psi$-scatter plot, shown in figure $15(d)$, has a linear relation for the core vortex and a nonlinear relation for the satellites, while the branch for $\omega=0$ represents the external flow. The $\omega, \psi$-relation was obtained after the transformation $\psi^{\prime}=\psi+\frac{1}{2} \Omega_{d i g} r^{2}$ and $\omega^{\prime}=\omega-2 \Omega_{\text {dig }}$, with $\Omega_{\text {dig }}$ the correction rotation speed of the tripole, so that $J\left(\omega^{\prime}, \psi^{\prime}\right)=0$ with respect to the co-rotating frame of reference. The $\omega, \psi$-relation shows a remarkable correspondence with the tripole scatter plot observed in numerical simulations of a forced two-dimensional turbulent flow by Legras, Santangelo \& Benzi (1988). Such well-defined scatter plots were obtained by iteratively changing the rotation speed $\Omega_{\text {dig }}$ until the size of the tripole vortices in the streamline pattern matched with that in the vorticity contours. Within the experimental error of $10 \%$ (which is mainly determined by the interpolation of the digitized velocity field onto a grid), this procedure yielded a value of $\Omega_{\text {dig }}$ that corresponded well with the rotation speed $\Omega_{\text {exp }}$ that was measured directly from the orientation angle of the structure in subsequent streak photographs, which is 

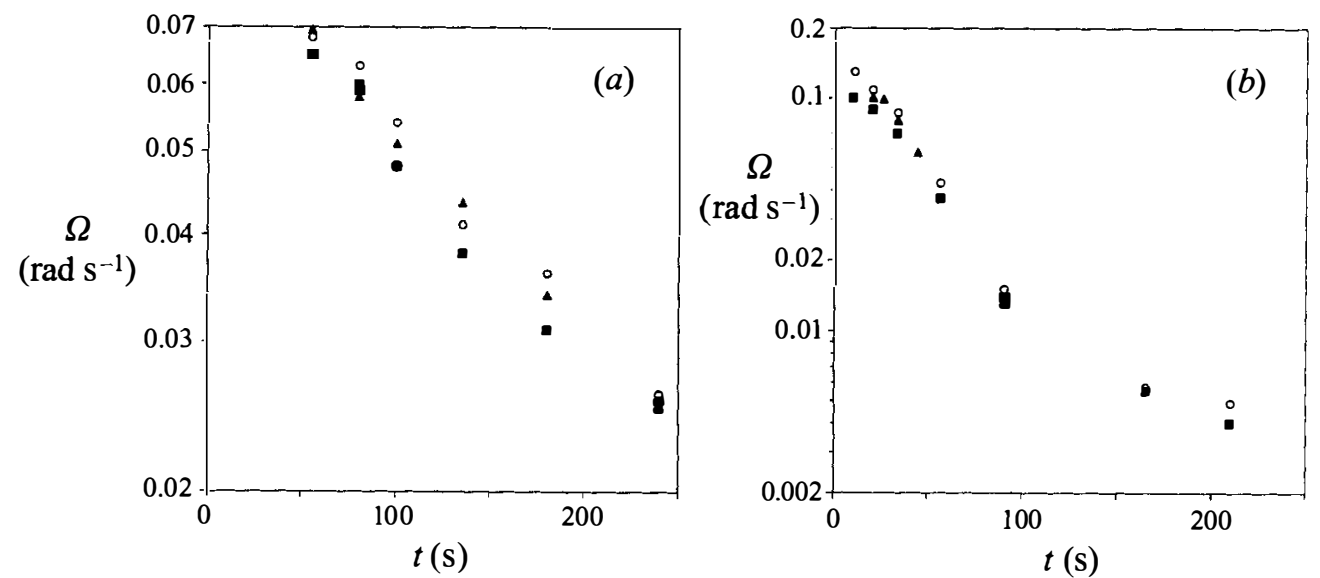

FIGURE 16. Graphical presentation of the rotation speeds of $(a)$ the tripolar vortex and $(b)$ the unstable tripolar vortex of figure 13, i.e. $\Omega_{\exp }(\boldsymbol{\Delta})$ as determined from consecutive streak photographs; the correction speed for $\omega, \psi$-scatter plots $\Omega_{\text {dig }}(\mathbf{Q})$, and according to the point-vortex model $\Omega_{\text {point }}$ $(\circ)$. Af ter approximately $t=150 \mathrm{~s}$ the unstable tripole $(b)$ has transformed into a triangular vortex, see also figure 19.

demonstrated graphically in figure $16(a)$. In this graph the vertical axis has a logarithmic scale so that an exponential decay is displayed by a straight line. Similar to the monopolar structures discussed in \$3.2.2, the tripolar structures reveal an approximately exponential decay.

The circulation of the core vortex $\left(\gamma_{c}\right)$ and that of the satellites $\left(\gamma_{s}\right)$ were calculated by numerically integrating the positive vorticity (representing the core vortex) and negative vorticity values (representing the satellites) separately over the digitized area $A$ that contained the vortex structure. For $N$ grid points the circulations $\gamma_{c}$ and $\gamma_{s}$ are given by

$$
\left.\begin{array}{l}
\gamma_{c}=\int_{A} \omega_{+} \mathrm{d} A=\sum_{1}^{N} \omega_{+} \Delta x \Delta y, \\
\gamma_{s}=\frac{1}{n} \int_{A} \omega_{-} \mathrm{d} A=\frac{1}{n} \sum_{1}^{N} \omega_{-} \Delta x \Delta y,
\end{array}\right\}
$$

where $n$ is the number of satellites and $\gamma_{s}$ thus represents the circulation averaged over the satellites. Because the circulation of the satellites was approximately equal in the cases studied here, the error introduced by taking the average was negligible. The separation distances between the satellites and the core vortex were measured from vorticity contour plots and averaged, so that $a=\left(a_{1}+a_{2}\right) / 2$ for the tripole and in case of a triangular vortex discussed in the next section $a=\left(a_{1}+a_{2}+a_{3}\right) / 3$.

The values for the circulations $\gamma_{s}$ and $\gamma_{c}$ are shown in figure $17(a)$ as a function of time. The graph shows the decay of the circulation of the core vortex and that of the satellites. In order to verify whether the net circulation remains zero, for each time $\gamma_{s}$ is plotted versus $\gamma_{c}$ in figure $17(b)$. Although the ratio between these values oscillates in time, the mean value appears to correspond quite well with the solid line $\gamma_{s}=-\gamma_{c} / 2$ that represents zero net circulation.

The separation distance $a=\left(a_{1}+a_{2}\right) / 2$ between the satellites, normalized with the initial separation distance $a_{0}$ of the tripole after its generation, increases approximately linearly in time. A least-square linear fit yielded a coefficient $5.9 \times 10^{-4} \mathrm{~s}^{-1}$, which 
17
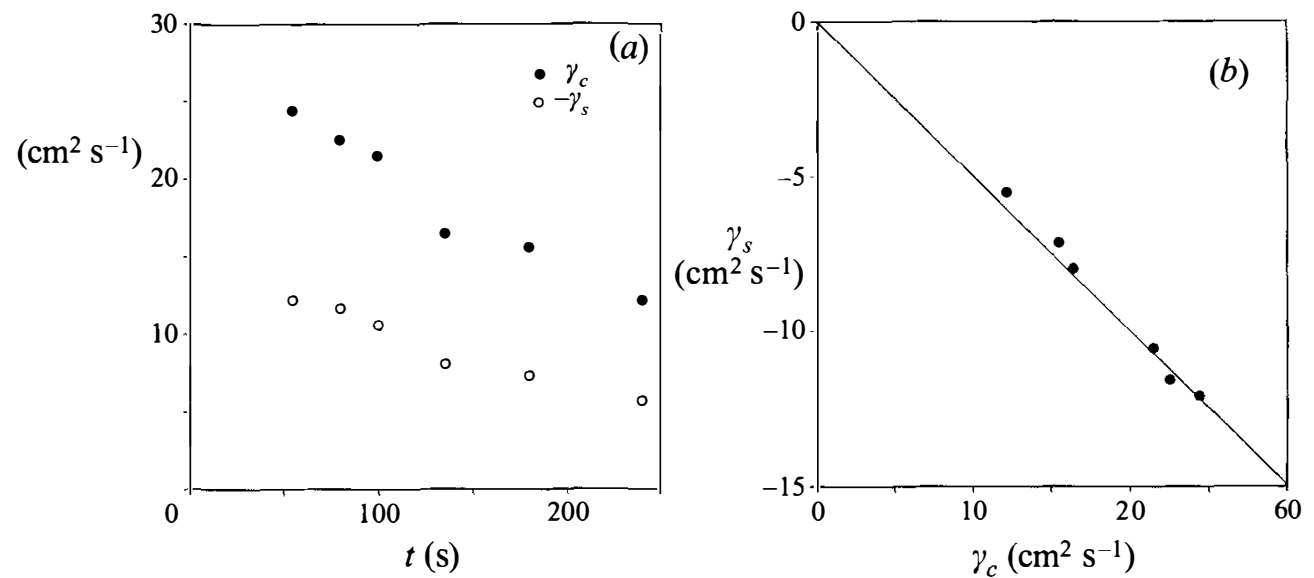

FigURE 17. Graphical presentation of $(a)$ the measured decay in circulation of the core vortex $\gamma_{c}$ $(\bullet)$ and that of the mean circulation of the satellites $-\gamma_{s}=-\left(\gamma_{1}+\gamma_{2}\right) / 2(0)$ of the tripolar vortex shown in figure 15; (b) the ratio of the two values compared with the line $\gamma_{c}=-2 \gamma_{s}$ that represents zero net circulation.
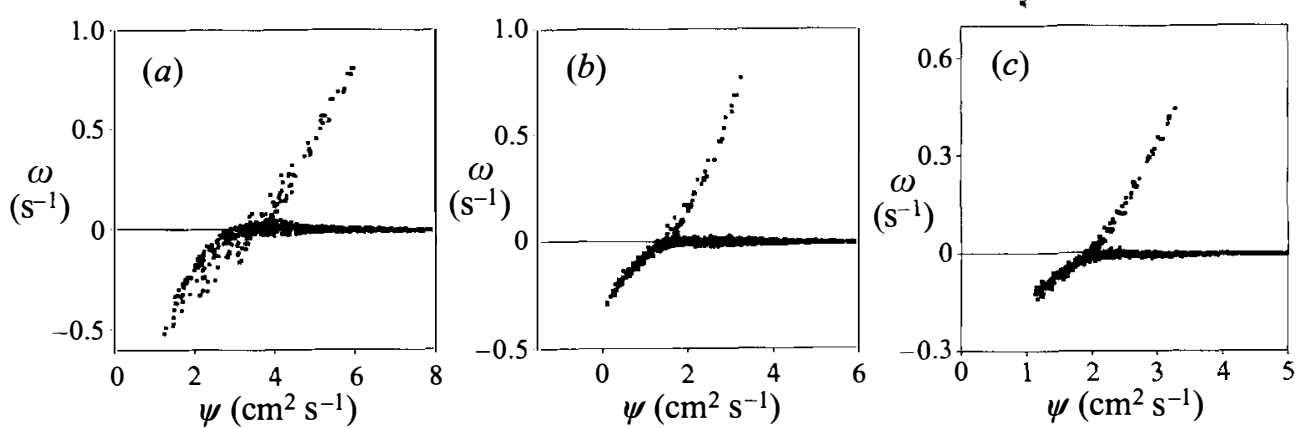

FIGURE 18. A sequence of $\omega, \psi$-scatter plots representing the evolution of the tripolar vortex at $t=55 \mathrm{~s}(a), 135 \mathrm{~s}(b)$ and $240 \mathrm{~s}(c)$. Experimental parameters: see figure 12.

corresponds with an increase of $14 \%$ in one rotation period of the tripole (because the tripole decayed relatively fast, only one rotation period has been measured). This dilatation of the tripole is due to entrainment of ambient fluid between the core vortex and the satellites. In rotating fluids the horizontal expansion rate also increased linearly in time (see van Heijst et al. 1991). During the first rotation period the growth rate of the tripole was, similarly, approximately $13 \%$, but, presumably due to viscous effects, increased during the further flow evolution.

The evolution of the tripole shown in figure 12 is illustrated by the sequence of scatter plots shown in figure 18. Although the core vortex conserves its characteristic linear $\omega, w$-relation, the negative branch - representing both satellites - changes in shape during the flow evolution. Initially, the negative branch shows some scatter and seems to contain two well-defined slightly separated negative branches (see figure $18 a$ ) indicating an asymmetry of the tripolar vortex which was due to the mis-alignment of the three vortex centres. However, at some later stage this asymmetry vanishes and eventually the $\omega, \psi$-relation of the negative branches becomes approximately linear (see figure $18 c$ ). Neglecting the initial nonlinearities of these branches, the positive branch and the negative branch have slightly different slopes and make an angle at the 

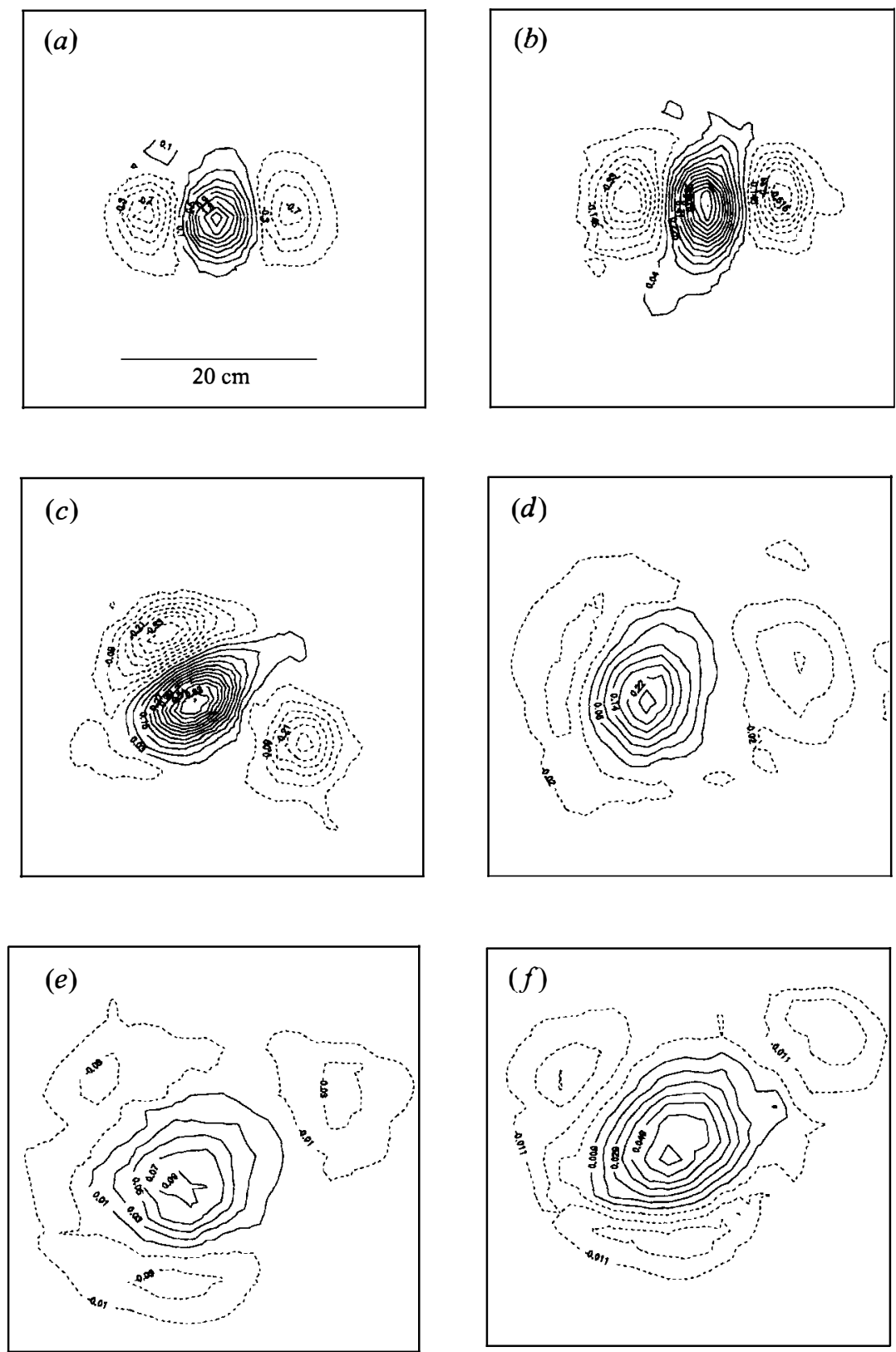

FigURE 19. Sequence of vorticity contour plots of the unstable tripole evolution, at $t=20 \mathrm{~s}(a)$; $33 \mathrm{~s}(b) ; 56 \mathrm{~s}(c) ; 90 \mathrm{~s}(d) ; 165 \mathrm{~s}(e)$ and $(f) 210 \mathrm{~s}$. Experimental parameters: see figure 13.

level $\omega=0$. With time, the difference in these slopes increases, and the $\omega, \psi$-relation eventually tends to that of a monopolar vortex as shown in figure $7(b)$. Therefore, it can be anticipated that this tripole will presumably also become unstable eventually and evolve into a monopolar vortex.

\subsection{The triangular vortex}

Figure 19 shows a sequence of vorticity contour plots of the experiment on the unstable tripole shown in figure 13 , which clearly reveal the evolution in the vortical 

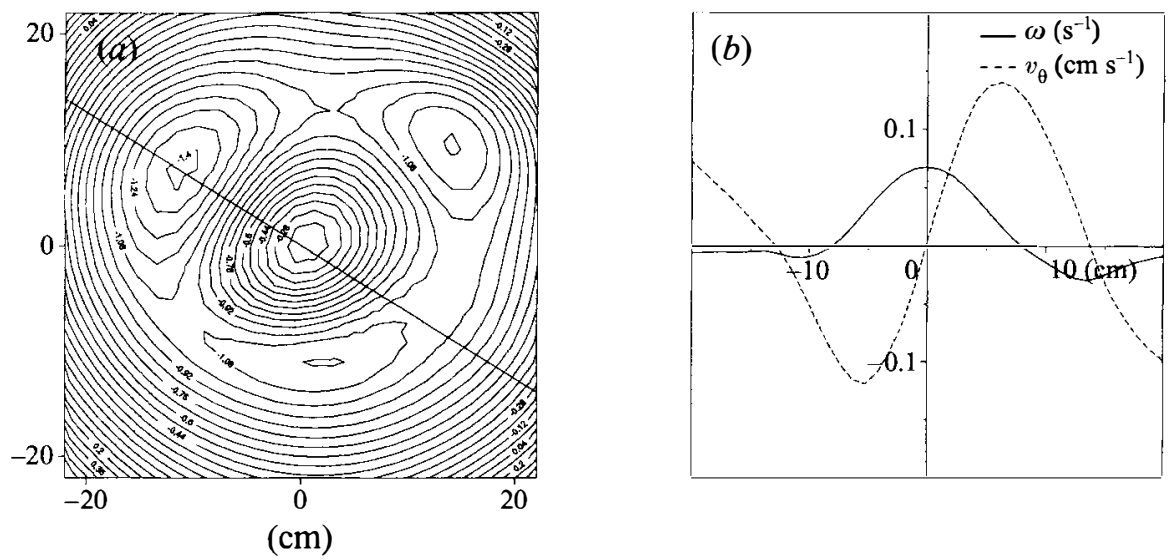

FIGURE 20. Contour plot of the stream function relative to the co-rotating frame of reference $(a)$, and $(b)$ a typical cross-sectional profile of the azimuthal velocity (dashed line) and the vorticity (solid line) along the straight line indicated in $(a)$.

flow. The initial tripolar vortex (figure $19 a, b$ ) has slightly asymmetrically aligned vortex centres. Owing to the smaller distance of the left-hand satellite vortex to the core, the core vortex forms a compact couple with that vortex (figure $19 \mathrm{c}$ ). In a subsequent stage after rotating anticlockwise over about $40^{\circ}$, the left-hand satellite vortex is deformed to an elongated patch that is torn into two separate satellites of approximately equal strength, thus eventually forming a triangular vortex (see figure $19 d, e)$. The deformation of the satellites is probably due to entrainment as well as viscous effects that cause a faster decay of the satellites (as will be discussed later in $\S 7)$. This triangular vortex did not show any significant changes in shape during the further decay (see figure $19 e, f$ ).

The triangular shape of the vortex structure is also clearly visible in the $\psi$-contour lines shown in figure $20(a)$. The vorticity and velocity profiles along a cross-section through two vortex centres, as indicated by the line in figure $20(a)$, are shown in figure $20(b)$, and reveal that this triangular vortex structure has a continuous vorticity distribution, similar to the dipolar and the tripolar vortex.

A sequence of $\omega, \psi$-scatter plots showing the evolution of the unstable tripole is presented in figure 21. Initially the structure reveals an approximately linear $\omega, \psi$ relation in the core vortex. The two separate nonlinear negative branches are due to the different angular velocities of the satellites. The instability of the structure results in the transport of vorticity, as can be observed from the two scatter regions for $\omega<0$ in the plot of figure $21(b)$, which indicates deformation of the satellites. In figure $21(c)$, the scatter in the negative branch is reduced and reflects three satellite vortices of a quasi-stationary triangular vortex. This triangular vortex has initially a slightly nonlinear $\omega, \psi$-relation that becomes linear in time (see figure $21 c, d$ ), however. The relatively large scatter in the $\omega, \psi$-plots is attributed to slight non-stationarities, as well as to the fact that the two separation distances were not equal, which increases the error in the correction rotation speed.

The correction rotation speeds $\Omega_{d i g}$ and $\Omega_{e x p}$ of this evolving unstable tripolar vortex are displayed graphically as a function of time in figure $16(b)$. Initially, the vortex structure displays an exponential decay, similar to the decay of the stable tripolar vortex. Owing to its transition to a triangular vortex structure, i.e. around $t=100 \mathrm{~s}$, however, the tripole rotates slower than according to an exponential 

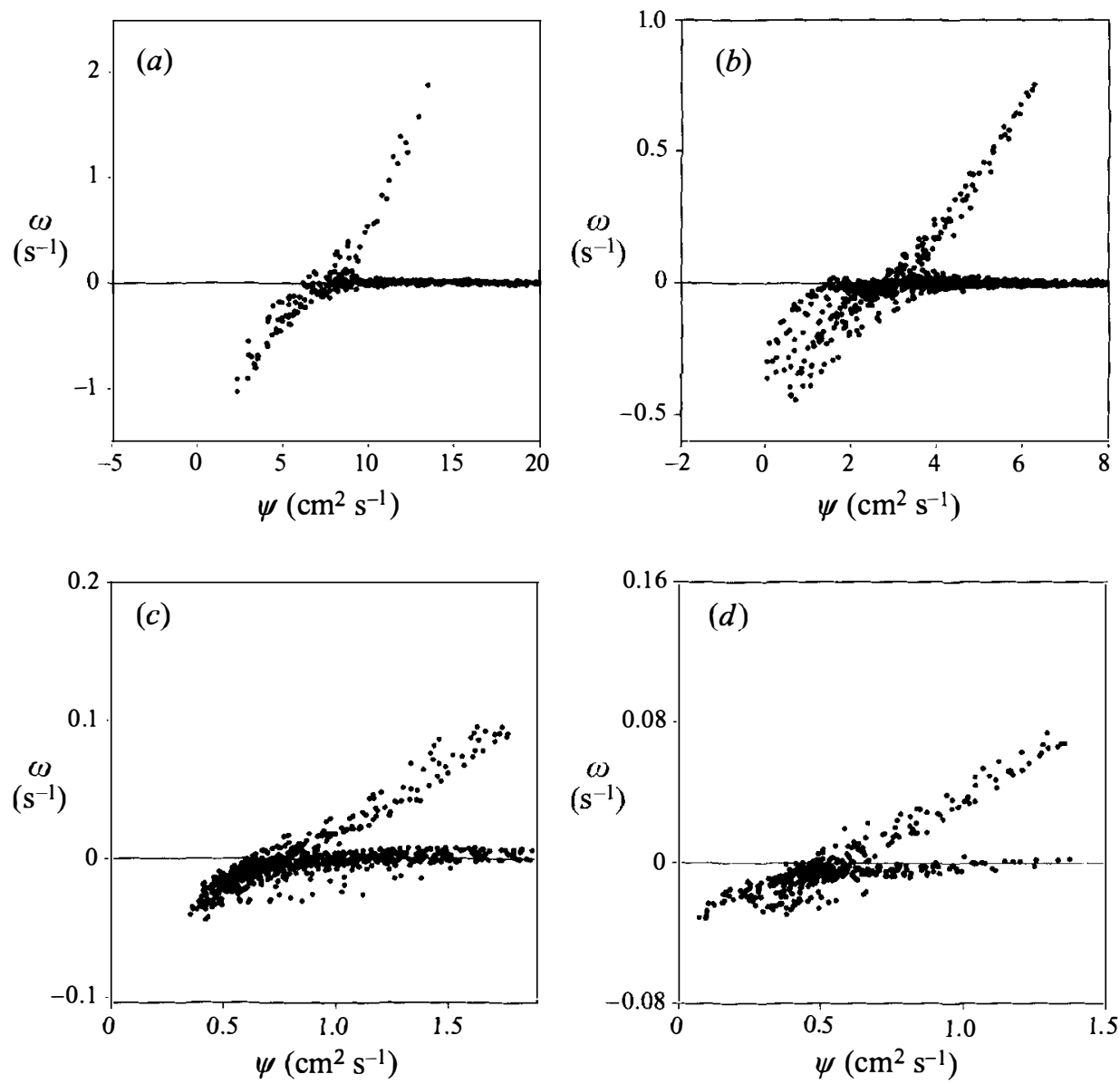

FIGURE 21. A sequence of $\omega, w$-scatter plots of an unstable tripole that resulted in the formation of a triangular vortex at $t=20 \mathrm{~s}(a) ; 56 \mathrm{~s}(b) ; 165 \mathrm{~s}(c)$ and $210 \mathrm{~s}(d)$ after forcing. Experimental parameters: see figure 13 .

decay. Unfortunately, sufficient data were not available to follow the decay of the triangular vortex. Because the structure rotated slowly during the transition, for all subsequent pictures a significant rotation speed could not be measured from the streak photographs.

Although this triangular vortex structure conserves its characteristic properties (triangular shape of the core, linear $\omega, \psi$-relation) during the course of the experiment, it might well be that the flow was too weak to develop instabilities. Some stability properties of multipoled structures in a stratified fluid are discussed further in $§ 7$.

\section{Point-vortex models}

For the tripolar vortex as well as the triangular vortex, no vortex models with a continuous vorticity distribution are at present known. However, a very useful representation of complex vortical flows can be given by a set of point vortices. Although such point vortices describe an irrotational velocity field, with the vorticity being concentrated in singular points, it has been shown in related studies that their motion as well as their advection of passive tracer particles can give insight in the 
(a)

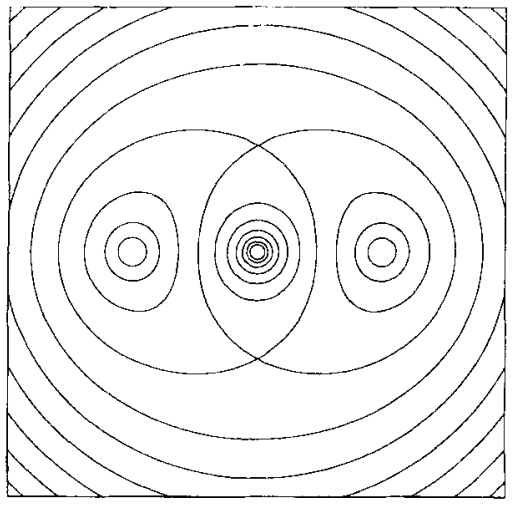

(b)

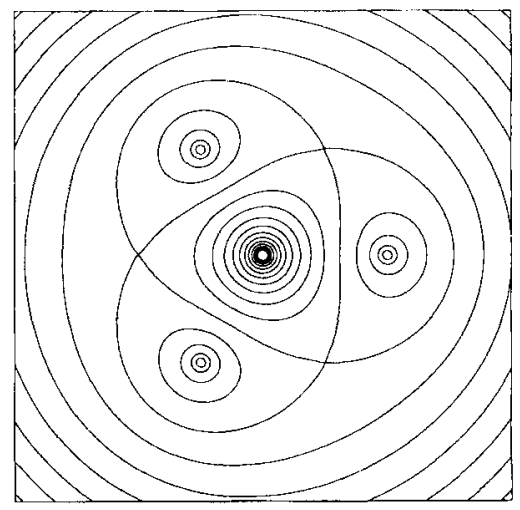

FIGURE 22. Isolines of the stream function relative to the co-rotating frame of reference of the tripolar vortex $(a)$ and the triangular vortex $(b)$ as modelled by point vortices according to the point-vortex models (6.3) and (6.5).

dynamics of complex vortex structures. This has for instance been demonstrated in an analytical-numerical study on dipolar vortices and vortex interactions by Meleshko et al. (1992), as well as in numerical simulations of the mutual interaction of large numbers of point vortices by Benzi et al. (1987). In related studies on vortices in rotating fluids by van Heijst et al. (1991) and by Kloosterziel (1990), tripolar vortices and triangular vortices were compared satisfactorily with point-vortex models. Pointvortex models for the tripolar and triangular vortices are briefly discussed below, and are subsequently compared with the observed laboratory vortices.

\subsection{Models}

A simple point-vortex model for an isolated tripolar vortex is represented by a linear, symmetric arrangement of three point vortices with strengths $\left(\gamma_{s}, \gamma_{c}, \gamma_{s}\right)=(-\gamma, 2 \gamma,-\gamma)$, with the satellites (of strength $\gamma_{s}$ ) at a distance $a$ from the central vortex. The stream function of this configuration is

$$
\psi=\frac{\gamma}{2 \pi a^{2}} \ln \left\{1+\frac{1-2\left(x^{2}-y^{2}\right)}{\left(x^{2}+y^{2}\right)^{2}}\right\}^{1 / 2},
$$

while the angular speed can be calculated from their mutually induced velocity and is given by the relation

$$
\Omega_{2}=\frac{3}{4}-\frac{\gamma}{\pi a^{2}} .
$$

With respect to a co-rotating frame of reference the stream function can be written as

$$
\psi_{2}=\frac{\gamma}{2 \pi a^{2}}\left[\ln \left\{1+\frac{1-2\left(x^{2}-y^{2}\right)}{\left(x^{2}+y^{2}\right)^{2}}\right\}^{1 / 2}+\frac{3}{4}\left(x^{2}+y^{2}\right)\right]
$$

and the corresponding streamline pattern is shown in figure 22(a), for $a=1$ and $\gamma=1$. Although the streamlines that enclose the tripole slightly differ in shape from the $\psi$-contour plot in figure $15(b)$, the main characteristics are represented by this model: the point-vortex configuration describes a rotational motion and it has three sets of nested closed streamlines.

An isolated triangular vortex can be simply modelled by four point vortices with 
strengths $\left(\gamma_{c}, \gamma_{s}, \gamma_{s}, \gamma_{s}\right)=(3 \gamma,-\gamma,-\gamma,-\gamma)$, where the satellites are located on a circle of radius $a$ around the central vortex, with an equal angular spacing of $2 \pi / 3$. The rotation speed for this configuration is

$$
\Omega_{3}=\frac{\gamma}{\pi a^{2}},
$$

so that in the co-rotating frame the stream function is given by

$$
\psi_{3}=\frac{\gamma}{2 \pi a^{2}}\left[\ln \left\{1+\frac{1-2 x\left(x^{2}-3 y^{2}\right)}{\left(x^{2}+y^{2}\right)^{3}}\right\}^{1 / 2}+\left(x^{2}+y^{2}\right)\right] .
$$

The corresponding streamline pattern is shown in figure 22(b). Again, the pointvortex model appears to represent the main features of the triangular vortex, i.e. the structure performs a rotational motion and consists of four sets of closed nested streamlines of which the core region has a triangular shape.

\subsection{Comparison with experimental results}

As a check of the validity of the tripolar point vortex model one can compare the experimental rotation speeds $\Omega_{\text {exp }}$ and $\Omega_{\text {dig }}$, with that predicted by the point-vortex model according to relation (6.2), where $\gamma$ is taken equal to $\left(\gamma_{c}-\gamma_{s}\right) / 3$; the results are shown graphically in figure 16(a). Note that for each instant $t$ at which a comparison is made, the point-vortex strengths and core-satellite separation distances were derived from the actual, measured flow field. Although the rotation speed of the point-vortex model is systematically larger than $\Omega_{\text {dig }}$ and $\Omega_{\text {exp }}$, the agreement is reasonable, taking into account the experimental error of $10 \%$ in these values. The systematic deviation is probably due to the estimated positioning of the point vortices.

Because the triangular vortex formed at a later stage of the evolution of an unstable tripolar vortex, only a few measurements of this triangular structure are available. The comparison between the experimental values $\Omega_{\text {exp }}$ and $\Omega_{\text {dig }}$ of the rotation speed and the point-vortex model prediction (6.4) is shown in figure $16(b)$. It should be kept in mind that this graph presents $\Omega$-values for a rotating structure that gradually transforms from a tripolar vortex into a triangular vortex; the formation of the triangular structure is completed approximately at $t=150 \mathrm{~s}$ (see also figure 19). As for the tripolar vortex $(t<150 \mathrm{~s})$, the measured rotation speed of the triangular vortex $(t>150 \mathrm{~s})$ corresponds fairly well with the value according to the point-vortex model.

\section{Conclusions and discussion}

Planar monopolar vortex structures in a stratified fluid have been investigated: stable monopoles as well as multipoled vortex structures that arise from unstable monopoles. Three different forcing methods were used to generate a monopolar vortex and, apart from the influence of the internal waves on the evolution of the vortex, similar characteristics were found for the stable monopolar vortices. The vorticity structure of the monopoles could be characterized by a single-signed core vortex surrounded by a large ring of relatively weak oppositely signed vorticity. The core of the vortex had an approximately linear $\omega, \psi$ relation. During the flow evolution, interaction with internal waves generated during the forcing caused entrainment of ambient fluid and led to a horizontal expansion of the vortex. Thereby, the vorticity ring around the core widened and its vorticity amplitude gradually decreased, while 
the velocity profile of the vortex became less steep. As a consequence, the stablility of the vortex increased during its evolution.

Three decay models are presented, with the Bessel-type vortex representing the basic flow, and the decay caused by (mainly vertical) diffusion of vertical vorticity. Although horizontal entrainment is neglected, the models show a reasonable agreement with the data. The dynamics of the present decaying vortices in a stratified fluid can be relevant to vortex lenses in the ocean for which the Burger number $S=N^{2} H^{2} /\left(f^{2} L^{2}\right) \gg 1$, with $L$ and $H$ the horizontal and the vertical length scales, respectively, and $f$ the rotation frequency. In that case Ekman circulation is suppressed and the decay is governed by vertical diffusion which leads to a thickening of the lens structure (Garrett 1982).

The monopoles were found to be stable for Froude numbers $F<0.1$, while they were unstable for $F>0.1$, in which case the formation of multipoled structures was observed. Tripolar or unstable triangular vortex structures were observed to form, while in some cases the vortex split up into two dipolar vortices, similar to the observations by Kloosterziel \& van Heijst (1991) and Griffiths \& Linden (1981). However, in contrast to the observations by Kloosterziel \& van Heijst (1991), here the emerging tripolar vortices became asymmetric in most cases owing to the interaction with internal waves.

Apart from dipole splitting, two different evolutions of the unstable tripole were observed: the tripolar vortex with weak satellites formed a monopole, while the tripole with strong satellites formed a persistent triangular vortex.

In the first case the satellites decayed faster owing to entrainment of ambient fluid and they were eventually deformed by the shear (induced by the core vortex) into filaments, so that a monopolar vortex emerged; in the latter case one of the satellites was observed to split into two vortex patches and the core vortex obtained a triangular shape, leading to the formation of a persistent triangular vortex. Since neither the tripolar vortex structure nor the triangular vortex structure have been observed before in a non-rotating stratified fluid, it was decided to study their characteristics in detail.

It was found that both vortex structures have a continuous vorticity distribution. The tripole has an $\omega, \psi$-relation that is linear for the core vortex and nonlinear for the satellites, similar to that found for the tripole in the numerical simulations by Legras et al. (1988). The triangular vortex has a linear $\omega, \psi$-relation, which is at variance with the numerical results of Orlandi \& van Heijst (1992); Carton (1992); Morel $\&$ Carton (1993) and Carnevale \& Kloosterziel (1994) who found an $\omega, \psi$-relation with $\partial \omega / \partial \psi=0$ in the central vortex core. Both vortices are compared with a point-vortex model that turned out to be very useful as an approximate description of these complicated vorticity structures.

Typically, the satellites of multipoled structures appeared to become relatively weaker than the core vortex due to entrainment of ambient fluid, thus leading to the eventual instability of the structure. This is at variance with the evolution of tripolar vortices in purely two-dimensional numerical simulations (see Carton et al. 1989; Orlandi \& van Heijst 1992) and in rotating homogeneous fluids (see Kloosterziel \& van Heijst 1991) and is apparently due to a three-dimensional effect. Owing to vertical diffusion of vorticity the structure obtains a vertical vorticity distribution with a certain thickness; vortical structures with a larger vertical thickness $(2 \sigma)$ decay slower than thin structures. However, there is good reason to believe that the present planar multipoled vortex structures have a spatially non-uniform thickness and thereby also decay locally faster. Such a variation in thickness may develop just after vortex generation, by diffusion of vorticity that smooths the vertical vorticity gradients over a certain thickness. Owing to the large vertical vorticity gradients in 
the vortex centre compared to the vorticity gradients at the edge the diffusion results in a lens-like vorticity structure, being thick in the centre and thin at the edges. This implies a faster decay of the (thinner) outer region. Moreover, by entrainment and the formation of satellite vortices, the vortex expands horizontally and 'intrudes' into ambient quiescent fluid. This results in relatively large vertical vorticity gradients at the upper and lower boundaries of the intruded vortex region, implying also a faster decay of the outer regions. As a consequence, the satellite vortices of planar vortex structures decay faster than the core, which implies a tendency to evolve to a monopolar vortex eventually.

In the present experiments, this instability mechanism had much more effect on the asymmetric tripoles because of their relatively large entrainment: by entrainment the vortex separation distances (see figure 19 ) increased approximately $60 \%$ during one rotation, compared to $13 \%$ for the symmetric tripole. This mechanism is very similar to the evolution of unstable lens-shaped vortices in rotating stratified fluids, where the transition between mode- 2 and mode- 3 vortices and the subsequent relaxation to the state of a monopolar vortex also occurred (see Griffiths \& Linden 1981): the satellite vortices were confined in a layer that is relatively thin compared to the core vortex (as was demonstrated by visualizations) and decayed relatively fast.

In summary, this study has shown that multipoled coherent vortex structures, containing net angular momentum, also occur in stratified fluids. Moreover, their properties are similar to those of their counterparts in purely two-dimensional flows. A major difference, however, lies in the long-term behaviour of the multipoled vortex structures: because in a stratified fluid the decay rate is highest in the satellite vortices (their thickness being smaller than that of the core vortex), these multipolar vortex structures are expected to relax to a monopolar vortex, eventually.

The authors are grateful to the students Robert van Sluis, Walter Govers and Eric de Hoog for their contributions in analysing some of the data of the experiments. One of us (J.B.F.) gratefully acknowledges financial support by the Foundation for Fundamental Research on Matter (FOM) of the Netherlands Organization for Pure Research (NWO).

\section{REFERENCES}

Batchelor, G. K. 1967 An Introduction to Fluid Dynamics. Cambridge University Press.

Benzi, R., Patarnello, S. \& Santangelo, P. 1987 On the statistical properties of two-dimensional decaying turbulence. Europhys. Lett. 3, 811-818.

Carnevale, G. F. \& Kloosterziel, R. C. 1994 Emergence and evolution of triangular vortices. J. Fluid Mech. 259, 305-331.

CARTON, X. J. 1992 On the merger of shielded vortices. Europhys. Lett. 18, 697-703.

Carton, X. J., Flierl, G. R. \& Polvani, L. M. 1989 The generation of tripoles from unstable axisymmetric isolated vortex structures. Europhys. Lett. 9, 339-344.

Flór, J. B. \& van Heisst, G. J. F. 1994 An experimental study on dipolar structures in a stratified fluid. J. Fluid Mech. 279, 101-133.

Flór, J. B., Heisst, G. J. F. van \& Delfos, R. 1995 Decay of dipolar structures in a stratified fluid. Phys. Fluids 7, 374-383.

FoRTUIN, J. M. H. 1960 Theory and application of two supplementary methods of constructing density gradient columns. J. Polymer Sci. 44, 505-515.

Garrett, C. 1982 On spindown in the ocean interior. J. Phys. Oceanogr. 12, 989-993.

GrifFITHS, R. W. \& LindEN, P. F. 1981 The stability of vortices in a rotating, stratified fluid. J. Fluid Mech. 105, 283-316. 
Hedström, K. \& ARMI, L. 1988 An experimental study of homogeneous lenses in a rotating stratified fluid. J. Fluid Mech. 191, 535-556.

Heisst, G. J. F. van \& Kloosterziel, R. C. 1989 Tripolar vortices in a rotating fluid. Nature 338, 569-571.

Heisst, G. J. F. van, Kloosterziel, R. C. \& Williams, C. W. M. 1991 Laboratory experiments on the tripolar vortex in a rotating fluid. J. Fluid Mech. 225, 301-331.

Hopfinger, E. J. \& Heisst, G. J. F. van 1993 Vortices in rotating fluids. Ann. Rev. Fluid Mech. 25, 241-289.

KLOOSTERZIEL, R. C. 1990 Barotropic vortices in a rotating fluid. PhD thesis, University of Utrecht, The Netherlands.

Kloosterziel, R. C. \& Heisst, G. J. F. van 1991 An experimental study of unstable barotropic vortices in a rotating fluid. J. Fluid Mech. 223, 1-24.

Kloosterziel, R. C. \& Heisst, G. J. F. van 1992 The evolution of stable barotropic vortices in a rotating free-surface fluid. J. Fluid Mech. 239, 607-629.

Legras, B., Santangelo, P. \& Benzi, R. 1988 High-resolution numerical experiments for forced two-dimensional turbulence. Europhys. Lett. 5, 37-42.

Lerth, C. E. 1984 Minimum enstrophy vortices. Phys. Fluids 27, 37-42.

Meleshio, V. V., Kostantinov, M. Yu., Gurzhi, A. A. \& Konovaljuk, T. P. 1992 Advection of a vortex pair atmosphere in a velocity field of point vortices. Phys. Fluids A 4, 2779-2797.

MOREL, Y. G. \& CARTON, X. J. 1994 Multipolar vortices in two-dimensional incompressible flows. J. Fluid Mech. 267, 23-51.

NGUyen Duc, J. M. \& Sommeria, J. 1988 Experimental characterization of steady two-dimensional vortex couples. J. Fluid Mech. 192, 175-192.

Orlandi, P. \& HeIJST, G. J. F. vAN 1992 Numerical simulation of tripolar vortices in 2D flow. Fluid Dyn. Res. 9, 179-206.

Riley, J. J., Metcalfe, R. W. \& Weissman, M. A. 1981 Direct numerical simulations of homogeneous turbulence in density stratified fluids. Proc. AIP Conf. Nonlinear Properties of Internal Waves (ed. B. J. West), pp. 79-112.

SAUNDERS, P. M. 1973 The instability of a baroclinic vortex. J. Phys. Oceanogr. 3, 61-65. 